\title{
Reconciliation of local and global symmetries for a class of crystals with defects
}

\author{
Gareth P. Parry · Rachel Sigrist
}

Received: date / Accepted: date

\begin{abstract}
We consider the symmetry of discrete and continuous crystal structures which are compatible with a given choice of dislocation density tensor. By introducing the notion of a 'defective point group' (determined by the dislocation density tensor), we generalize the notion of Ericksen-Pitteri neighbourhoods to this context.
\end{abstract}

Keywords Crystals $\cdot$ Defects $\cdot$ Lie groups

Mathematics Subject Classification (2000) MSC 74A20 - MSC 74E25

\section{Introduction}

The purpose of this paper is to generalize, to the case of crystals with certain uniform distributions of defects, symmetry considerations which are well known in the case of perfect crystals. In a perfect crystal, geometrical considerations begin with the specification of basis vectors $\boldsymbol{e}_{1}, \boldsymbol{e}_{2}, \boldsymbol{e}_{3} \in \mathbb{R}^{3}$ of a perfect lattice

$$
L=\left\{\boldsymbol{x}: \boldsymbol{x}=n_{i} \boldsymbol{e}_{i}, n_{i} \in \mathbb{Z}, i=1,2,3\right\}
$$

- in a defective crystal, if one is given a set of three linearly independent vectors $\boldsymbol{e}_{1}, \boldsymbol{e}_{2}, \boldsymbol{e}_{3} \in \mathbb{R}^{3}$ and a dislocation density tensor $S$ (and this is what we shall be given), the first question is what set of points should be taken in generalization of the perfect lattice $L$.

Then, it is well known that the geometrical 'symmetries' of a perfect crystal structure relate to the various changes of basis that preserve the lattice $L$, and this leads to the requirement that, if $w(\cdot)$ is the continuum strain energy density function per unit current volume, then $w\left(\left\{\boldsymbol{e}_{i}\right\}\right)=w\left(\left\{\boldsymbol{e}_{i}^{\prime}\right\}\right)$ if $\boldsymbol{e}_{1}, \boldsymbol{e}_{2}, \boldsymbol{e}_{3}$ and $\boldsymbol{e}_{1}^{\prime}, \boldsymbol{e}_{2}^{\prime}, \boldsymbol{e}_{3}^{\prime}$ are different bases of the lattice $L$,

School of Mathematical Sciences, University of Nottingham, Nottingham, UK

Tel.: +44-(0)115-9513845

Fax: +44-(0)115-9513837

E-mail: gareth.parry@nottingham.ac.uk 
- we discuss this type of issue for defective crystals (of a certain class), having decided that the appropriate generalization of the perfect lattices is a set of points $G_{\ell}$. We will require that $w\left(\left\{\boldsymbol{e}_{i}\right\}, S\right)=w\left(\left\{\boldsymbol{e}_{i}^{\prime}\right\}, S^{\prime}\right)$ in the case that $\left(\left\{\boldsymbol{e}_{i}\right\}, S\right)$ and $\left(\left\{\boldsymbol{e}_{i}^{\prime}\right\}, S^{\prime}\right)$ generate the same set of points $G_{\ell}$. So, having recalled from Cermelli and Parry [5], Parry [12], [13] how to determine this set, the paper will be concerned with deriving the set of all geometrical quantities $\left(\left\{\boldsymbol{e}_{i}^{\prime}\right\}, S^{\prime}\right)$ which lead to a given $G_{\ell}$. This will end with a generalization, to crystals with defects, of the well known fact that $\left\{\boldsymbol{e}_{i}\right\}$ and $\left\{\boldsymbol{e}_{i}^{\prime}\right\}$ are bases of the same lattice $L$ if and only if $\boldsymbol{e}_{i}=\gamma_{i j} \boldsymbol{e}_{j}^{\prime}$ where $\gamma_{i j}$ are the integer components of a matrix $\gamma \in G L_{3}(\mathbb{Z})$.

We shall be concerned, also, with a generalization of the following result for a perfect crystal: let $C$ be a symmetric matrix with entries $\boldsymbol{e}_{i} \cdot \boldsymbol{e}_{j}, i, j=1,2,3$, then a frame indifferent strain energy function has the symmetry $w(C)=w\left(\gamma C \gamma^{T}\right)$, where $\gamma^{T}$ is the transpose of $\gamma$. Pitteri [17] has discussed the distribution of the points $\gamma C \gamma^{T}$ in the space of strictly positive definite symmetric matrices by showing that :

(i) the set $P(C)$ of matrices $\gamma \in G L_{3}(\mathbb{Z})$ such that $\gamma C \gamma^{T}=C$ is finite;

(ii) if $C_{0}$ is prescribed, then there is a neighbourhood $N\left(C_{0}\right)$ of $C_{0}$ such that if $C \in$ $N\left(C_{0}\right)$ then $\gamma C \gamma^{T} \in N\left(C_{0}\right)$ if and only if $\gamma \in P\left(C_{0}\right)$.

This result allows one to confine attention to a finite set of symmetries of the strain energy function, if one is concerned only with small but finite changes in the crystal configuration that is specified by $C_{0}$ - we shall provide a similar result for crystals with defects (in a given class).

The context of the paper is a continuum model of defective crystals proposed by Davini [6]. In that model the geometrical structure of the defective crystalline continuum is given by the prescription of three smooth linearly independent lattice vector fields $\ell_{1}(\cdot), \ell_{2}(\cdot), \ell_{3}(\cdot)$, defined at all points of a region $\Omega$ (in this paper we shall take $\Omega \equiv \mathbb{R}^{3}$ throughout). These lattice vector fields have duals $\boldsymbol{d}_{1}(\cdot), \boldsymbol{d}_{2}(\cdot), \boldsymbol{d}_{3}(\cdot)$, so that $\boldsymbol{d}_{a}(\boldsymbol{x}) \cdot \boldsymbol{\ell}_{b}(\boldsymbol{x})=\delta_{a b}, a, b=1,2,3,\left(\delta_{a b}\right)$ the Krönecker delta, $\boldsymbol{x} \in \mathbb{R}^{3}$, and the corresponding dislocation density tensor (ddt) $S$ is defined by

$$
S=\left(S_{a b}\right)=\frac{\nabla \wedge \boldsymbol{d}_{a} \cdot \boldsymbol{d}_{b}}{\boldsymbol{d}_{1} \cdot \boldsymbol{d}_{2} \wedge \boldsymbol{d}_{3}}, \quad a, b=1,2,3 .
$$

The ddt, $S$, is related to the Lie bracket of pairs of lattice vector fields, Parry and $\breve{S}$ ilhavý [11], and if $S \neq 0$ the vector fields are not commutative.

Note that in a perfect crystal there is a natural concept of the 'neighbour' of a given point: both $\boldsymbol{x}+\boldsymbol{e}_{a}$ and $\boldsymbol{x}-\boldsymbol{e}_{a}$ may be called neighbours of a given point $\boldsymbol{x} \in L$ relative to the vector field $\boldsymbol{\ell}_{a}(\cdot)$ defined by $\boldsymbol{\ell}_{a}(\boldsymbol{p})=\boldsymbol{e}_{a}, \boldsymbol{p} \in \mathbb{R}^{3}$. Said differently, if $\boldsymbol{x}(t)$ is the solution of $\dot{\boldsymbol{x}}=\boldsymbol{\ell}_{a}(\boldsymbol{x}(t))$, where the dot represents differentiation with respect to $t \in \mathbb{R}$, then $\boldsymbol{y}$ is called a neighbour of $\boldsymbol{x}$ (relative to the vector field $\boldsymbol{\ell}_{a}(\cdot)$ ) if either $(\boldsymbol{x}(0)=\boldsymbol{x}$ and $\boldsymbol{x}(1)=\boldsymbol{y})$ or $(\boldsymbol{x}(0)=\boldsymbol{y}$ and $\boldsymbol{x}(1)=\boldsymbol{x})$. The lattice $L$ is the set of points which consists of the origin, the neighbours of the origin (relative to each of the vector fields $\left.\ell_{1}(\cdot), \ell_{2}(\cdot), \ell_{3}(\cdot)\right)$, the neighbours of those neighbours, and so forth. The 'neighbour' idea extends to arbitrary vector fields $\ell_{a}(\cdot), a=1,2,3$, in the obvious way, irrespective of whether or not the ddt is zero, and the sets of points $G_{\ell}$ that we will adopt as generalizations of the perfect lattices will be precisely those sets of points which consist of the origin, its neighbours, the neighbours of those neighbours, etc., relative to prescribed lattice vector fields $\ell_{1}(\cdot), \ell_{2}(\cdot), \ell_{3}(\cdot)$. 
One aim of the paper is to discuss properties of energy functions of the form $w\left(\left\{\boldsymbol{e}_{i}\right\}, S\right)$ by associating a structure (i.e. a set of points in $\left.\mathbb{R}^{3}\right) G_{\ell}$ with the prescribed values of the geometrical variables $\left\{\boldsymbol{e}_{i}\right\}, S$. In constructing $G_{\ell}$, then, one has only the single prescribed value of $S$ (so far as the distribution of values of $S(\cdot)$ defined by (1) is concerned). To proceed, we assume that $S(\cdot)$ (defined by (1)) is constant in $\mathbb{R}^{3}$, with value equal to that prescribed as the second argument of $w$. (This would not be an appropriate assumption if $w$ were given to depend on gradients of $S$ also). Since $S$ involves derivatives of $\boldsymbol{d}_{a}(\cdot)$ ( or $\boldsymbol{\ell}_{a}(\cdot)$ ), one cannot make analogous assumptions regarding $\ell_{a}(\cdot)$, if $S \neq 0$. It is a main result in Lie theory (phrased rather differently to the following), that if $S(\cdot)$ is constant, then the neighbours of the origin are elements of a Lie group, and the subgroup of that Lie group which is generated by the neighbours of the origin is the set $G_{\ell}$ which consists of the origin, its neighbours, the neighbours of those neighbours, and so on. These facts are recalled in more detail in Parry [12], [13], and recalled briefly in section 2.1. One has also that:

(iii) Once it is assumed that $S(\cdot)$ is constant, there is defined a Lie group composition function $\psi(\cdot, \cdot): \mathbb{R}^{3} \times \mathbb{R}^{3} \rightarrow \mathbb{R}^{3}$ such that

$$
\boldsymbol{\ell}(\boldsymbol{\psi}(\boldsymbol{x}, \boldsymbol{y}))=\nabla_{1} \boldsymbol{\psi}(\boldsymbol{x}, \boldsymbol{y}) \boldsymbol{\ell}_{a}(\boldsymbol{x}), \quad a=1,2,3 .
$$

When (2) holds, $\ell_{a}(\cdot)$ is called a right invariant field with respect to the group multiplication function $\boldsymbol{\psi}(\cdot, \cdot)$. Putting $\boldsymbol{u}(\boldsymbol{x}) \equiv \boldsymbol{\psi}(\boldsymbol{x}, \boldsymbol{y})$ in (2), that equation can be rewritten as

$$
\boldsymbol{\ell}_{a}(\boldsymbol{u}(\boldsymbol{x}))=\nabla \boldsymbol{u}(\boldsymbol{x}) \boldsymbol{\ell}_{a}(\boldsymbol{x}), \quad a=1,2,3,
$$

which expresses an (elastic) self-similarity of the geometrical crystal configuration defined by the lattice vector fields. (In the case $S=0$, the elastic deformation $\boldsymbol{u}(\cdot)$ can be chosen to be a translation, and the fields become translation invariant);

(iv) It is shown in Davini [6] that $S$ is an elastic invariant, which leads to a certain arbitrariness in the choice of the corresponding Lie group (e.g. if $S=0$, then $\boldsymbol{d}_{a}(\cdot)$ may be any fields of the form $\boldsymbol{d}_{a}(\boldsymbol{x})=\nabla \psi_{a}(\boldsymbol{x})$, for potentials $\psi_{1}, \psi_{2}, \psi_{3}$ such that $\nabla \psi_{1} \wedge \nabla \psi_{2} \cdot \nabla \psi_{3} \neq 0$. A certain elastic deformation of the fields 'straightens' them out to $\left.\boldsymbol{d}_{a}(\cdot) \equiv \boldsymbol{e}_{a}\right)$. It is shown in Mal'cev [10], Parry [14], that this freedom allows one to choose the Lie group, in a canonical way, so that the integral curves through the origin of the lattice vector fields are straight lines (even when $S \neq 0$ );

(v) The structures $G_{\ell}$ that are generated when the Lie group is chosen in this canonical way are generally not discrete sets of points, but it is shown in Mal'cev [10], Thurston [18], Cermelli and Parry [5] that one can provide sufficient conditions on $S$ in order that these sets of points are discrete. (These are rationality conditions on a form of $S$ );

(vi) Mal'cev [10] provides a canonical form for the discrete set of points $G_{\ell}$, and shows that the automorphisms of $G_{\ell}$ (which are 'symmetries' of $G_{\ell}$ ) extend to automorphisms of the ambient Lie group;

(vii) Adopting the rationality conditions above, Cermelli and Parry [5] have investigated the nature of the structures $G_{\ell}$, and shown that they are multilattices in the sense of Ericksen [7] and Pitteri and Zanzotto [17].

The plan of the paper is as follows. In the next section we recall basic definitions and facts regarding Lie groups and algebras which will be useful later. These facts give information about $G_{\ell}$ as discrete subgroups of certain Lie groups - we generalize the relation $\boldsymbol{e}_{i}^{\prime}=\gamma_{i j} \boldsymbol{e}_{j}$ (which connects bases of a perfect lattice) to connect different 
(multiplicative) generators of the discrete subgroups $G_{\ell}$ via some linear transformation. The canonical choice of Lie groups helps here because it ensures that the one parameter subgroups of the canonical group are straight lines through the origin. To get at this connection, we also introduce the translation subgroup $T_{\ell}$ of $G_{\ell}$ - this is the set of vectors $\boldsymbol{t} \in \mathbb{R}^{3}$ such that $\boldsymbol{g}+\boldsymbol{t} \in G_{\ell}$ if $\boldsymbol{g} \in G_{\ell}$. $T_{\ell}$ has the structure of a perfect lattice, and one can introduce the linear transformations which map to $T_{\ell}$ to itself as a step on the way to describing the mappings from one set of (multiplicative) generators of $G_{\ell}$ to another. We also account for different descriptions of the cosets $G_{\ell} / T_{\ell}$ (represented by the 'shift' vectors in this particular example of a multilattice, in Ericksen's [7] and Pitteri and Zanzotto's [17] terminology).

We give, in Theorem 7 , necessary and sufficient conditions that different generators $\left\{\boldsymbol{e}_{i}^{\prime}\right\},\left\{\boldsymbol{e}_{i}\right\}$ give the same structure $G_{\ell}$, within the given canonical Lie group. It turns out that this canonical group is parameterized by an integer $k$ (which is related to the ddt, $S$ ), so we ask if the set of points corresponding to $G_{\ell}$ can be the same in different canonical groups (corresponding to different values of $k$, different values of $S)$. We accept that $w\left(\left\{\boldsymbol{e}_{a}^{\prime}\right\}, S^{\prime}\right)=w\left(\left\{\boldsymbol{e}_{a}\right\}, S\right)$ whenever the set of points generated by $\left\{\boldsymbol{e}_{a}^{\prime}\right\}, S^{\prime}$ is the same as the set of points generated by $\left\{\boldsymbol{e}_{a}\right\}, S$ (irrespective of the group structure), and calculate $\left(\left\{\boldsymbol{e}_{a}^{\prime}\right\}, S^{\prime}\right)$ in terms of $\left(\left\{\boldsymbol{e}_{a}\right\}, S\right)$. Finally we discuss whether or not there is a result analogous to that of Pitteri [16], in this case, to facilitate the analysis of small but finite changes of geometrical configuration in this class of discrete defective crystals.

\section{Definitions and preliminary results}

Here we recall relevant definitions and facts to do with Lie groups and Lie algebras, and make a particular choice of Lie group (given the value of the elastic invariant dislocation density tensor $S$ ). The discrete structures with which we shall be concerned are discrete subgroups of the chosen 'canonical' group $J$, and we prove a few preliminary results regarding the translation group associated with such a discrete subgroup in order to facilitate the discussion of symmetries of the subgroup in terms of linear transformations of $\mathbb{R}^{3}$ to itself, in the next section.

\subsection{Definitions}

A Lie group $G$ is a group with the structure of a manifold where the group multiplication function $\boldsymbol{\psi}: G \times G \rightarrow G$ is smooth. Group multiplication of elements $\boldsymbol{x}, \boldsymbol{y} \in G$ will be denoted here by $\boldsymbol{x} \boldsymbol{y} \equiv \boldsymbol{\psi}(\boldsymbol{x}, \boldsymbol{y})$. It will be sufficient for our purposes to identify elements of the group with points of $\mathbb{R}^{3}$, so that one may associate coordinates $x_{1}, x_{2}, x_{3} \in \mathbb{R}$ with a group element $\boldsymbol{x}=x_{i} \boldsymbol{e}_{i}$, by introducing a basis $\boldsymbol{e}_{1}, \boldsymbol{e}_{2}, \boldsymbol{e}_{3}$ of $\mathbb{R}^{3}$. Note that summation convention operates throughout, except when a summation is explicit. The group identity will be $\mathbf{0} \in \mathbb{R}^{3}$, and so

$$
\mathbf{0 x}=\boldsymbol{x} \mathbf{0}=\boldsymbol{x}, \quad(\boldsymbol{x y}) \boldsymbol{z}=\boldsymbol{x}(\boldsymbol{y} \boldsymbol{z}), \quad \boldsymbol{x} \boldsymbol{x}^{-1}=\boldsymbol{x}^{-1} \boldsymbol{x}=\mathbf{0},
$$

for all $\boldsymbol{x}, \boldsymbol{y}, \boldsymbol{z} \in G$, and where the inverse element $\boldsymbol{x}^{-1}$ of $\boldsymbol{x}$ exists for all $\boldsymbol{x} \in G$. 
The structure constants are defined to be the quantities

$$
C_{i j k}=\frac{\partial^{2} \psi_{i}}{\partial x_{j} \partial y_{k}}(\mathbf{0}, \mathbf{0})-\frac{\partial^{2} \psi_{i}}{\partial x_{k} \partial y_{j}}(\mathbf{0}, \mathbf{0}),
$$

where $\boldsymbol{\psi}(\boldsymbol{x}, \boldsymbol{y})=\psi_{i}(\boldsymbol{x}, \boldsymbol{y}) \boldsymbol{e}_{i}$, and the Lie bracket operation $[\cdot, \cdot]: \mathbb{R}^{3} \times \mathbb{R}^{3} \rightarrow \mathbb{R}^{3}$ is defined by

$$
[\boldsymbol{x}, \boldsymbol{y}]=C_{i j k} x_{j} y_{k} \boldsymbol{e}_{i}, \quad \boldsymbol{x}, \boldsymbol{y} \in \mathbb{R}^{3} .
$$

Here, the vector space $\mathbb{R}^{3}$ and the operation $[\cdot, \cdot]$ make up the Lie algebra which corresponds to the group $G$. Note that

$$
\left[\boldsymbol{e}_{i}, \boldsymbol{e}_{j}\right]=C_{k i j} \boldsymbol{e}_{k}
$$

Suppose that lattice vector fields $\boldsymbol{\ell}_{a}(\cdot), a=1,2,3$, have duals $\boldsymbol{d}_{a}(\cdot), a=1,2,3$, and define the dislocation density tensor $S=\left(S_{a b}\right)$ by

$$
S_{a b}(\boldsymbol{x})=\frac{\nabla \wedge \boldsymbol{d}_{a}(\boldsymbol{x}) \cdot \boldsymbol{d}_{b}(\boldsymbol{x})}{\boldsymbol{d}_{1}(\boldsymbol{x}) \cdot \boldsymbol{d}_{2}(\boldsymbol{x}) \wedge \boldsymbol{d}_{3}(\boldsymbol{x})}
$$

Then if $S_{a b}(\cdot)$ is constant for each $a, b=1,2,3$, there exists a Lie group $G$ such that $\left(S_{a b}\right)$ is related to the structure constants of $G$ with respect to $e_{1} \equiv \ell_{1}(\mathbf{0}), e_{2} \equiv$ $\ell_{2}(\mathbf{0}), \boldsymbol{e}_{3} \equiv \boldsymbol{\ell}_{3}(\mathbf{0})$ as basis via

$$
C_{k i j}=\varepsilon_{r i j} S_{k r}
$$

It follows, when $S=\left(S_{a b}(\cdot)\right)$ is constant, that the lattice vector fields $\boldsymbol{\ell}_{a}(\cdot), a=1,2,3$, are right invariant with respect to the composition function $\psi$, in the sense that

$$
\boldsymbol{\ell}_{a}(\boldsymbol{\psi}(\boldsymbol{x}, \boldsymbol{y}))=\nabla_{1} \boldsymbol{\psi}(\boldsymbol{x}, \boldsymbol{y}) \boldsymbol{\ell}_{a}(\boldsymbol{x}), \quad a=1,2,3
$$

where

$$
\nabla_{1} \boldsymbol{\psi}(\boldsymbol{x}, \boldsymbol{y}) \equiv \frac{\partial \boldsymbol{\psi}}{\partial \boldsymbol{x}}(\boldsymbol{x}, \boldsymbol{y})
$$

This is a self similarity property of lattice vector fields with constant $S$ (a generalization of the translational invariance of lattice vector fields when $S=0, \boldsymbol{\psi}(\boldsymbol{x}, \boldsymbol{y}) \equiv \boldsymbol{x}+\boldsymbol{y})$. If $\lambda(\cdot)$ is any right invariant field, satisfying

$$
\boldsymbol{\lambda}(\boldsymbol{\psi}(\boldsymbol{x}, \boldsymbol{y}))=\nabla_{1} \boldsymbol{\psi}(\boldsymbol{x}, \boldsymbol{y}) \boldsymbol{\lambda}(\boldsymbol{x})
$$

then

$$
\boldsymbol{\lambda}(\boldsymbol{x})=\nabla_{1} \boldsymbol{\psi}(\mathbf{0}, \boldsymbol{x}) \boldsymbol{\lambda}(\mathbf{0}),
$$

so that the field $\boldsymbol{\lambda}(\cdot)$ is determined by its value at the origin (once $\boldsymbol{\psi}$ is given). The integral curve through $\boldsymbol{x}_{0},\{\boldsymbol{x}(t) ; t \in \mathbb{R}\}$, of the right invariant field $\boldsymbol{\lambda}(\cdot)$ is defined to be the solution of

$$
\frac{d \boldsymbol{x}}{d t}=\boldsymbol{\lambda}(\boldsymbol{x}(t)), \quad \boldsymbol{x}(0)=\boldsymbol{x}_{0}, \quad t \in \mathbb{R} .
$$

Integral curves through the origin are one parameter subgroups of $G$, that is, the solutions of (13) satisfy $\boldsymbol{x}(t) \boldsymbol{x}(s)=\boldsymbol{x}(t+s), s, t \in \mathbb{R}$. The exponential mapping $\exp (t \boldsymbol{\lambda}): \mathbb{R}^{3} \rightarrow \mathbb{R}^{3}, t \in \mathbb{R}, \boldsymbol{\lambda} \in \mathbb{R}^{3}$, is defined by constructing the right invariant field $\boldsymbol{\lambda}(\cdot)$ from (12) and (13) by putting $\boldsymbol{\lambda}(\mathbf{0})=\boldsymbol{\lambda}$, setting

$$
\exp (t \boldsymbol{\lambda})\left(\boldsymbol{x}_{0}\right)=\boldsymbol{x}(t),
$$


and noting that $\exp (t \boldsymbol{\lambda})=\exp \left(t^{\prime} \boldsymbol{\lambda}^{\prime}\right)$, if $t \boldsymbol{\lambda}=t^{\prime} \boldsymbol{\lambda}^{\prime}$. Group elements $\mathrm{e}^{(t \boldsymbol{\lambda})}$ are defined by

$$
\mathrm{e}^{(t \boldsymbol{\lambda})}=\exp (t \boldsymbol{\lambda})(\mathbf{0})
$$

and it is a fact that

$$
\boldsymbol{\psi}\left(\mathrm{e}^{(t \boldsymbol{\lambda})}, \boldsymbol{x}\right) \equiv \mathrm{e}^{(t \boldsymbol{\lambda})} \boldsymbol{x}=\exp (t \boldsymbol{\lambda})(\boldsymbol{x}) .
$$

This, (16), is an important result which relates group multiplication to flow along the right invariant field. Let $\boldsymbol{x}_{0} \in G$ be given, and say that $\boldsymbol{y}$ is a neighbour of $\boldsymbol{x}_{0}$ if there is an index $a \in\{1,2,3\}$ such that either $\left(\dot{\boldsymbol{x}}=\boldsymbol{\ell}_{a}(\boldsymbol{x}), \boldsymbol{x}(0)=\boldsymbol{x}_{0}, \boldsymbol{x}(1)=\boldsymbol{y}\right)$ or $\left(\dot{\boldsymbol{x}}=\boldsymbol{\ell}_{a}(\boldsymbol{x}), \boldsymbol{x}(0)=\boldsymbol{y}, \boldsymbol{x}(1)=\boldsymbol{x}_{0}\right)$. Then, from (16), the subgroup $G_{\ell}$ of $G$ that is generated by the three elements $\mathrm{e}^{\left(\ell_{1}\right)}, \mathrm{e}^{\left(\ell_{2}\right)}, \mathrm{e}^{\left(\ell_{3}\right)}$, where $\ell_{1}=\ell_{1}(0)$, etc., is the set which consists of the origin, the neighbours of the origin, the neighbours of those neighbours, and so on.

An automorphism of the Lie algebra determined by the Lie bracket $[\cdot, \cdot]$ is here an invertible linear transformation $L: \mathbb{R}^{3} \rightarrow \mathbb{R}^{3}$ such that $[L \boldsymbol{x}, L \boldsymbol{y}]=L[\boldsymbol{x}, \boldsymbol{y}], \boldsymbol{x}, \boldsymbol{y} \in \mathbb{R}^{3}$ and the corresponding structure constants satisfy $C_{i j k} L_{j p} L_{k q}=L_{i r} C_{r p q}$. An automorphism of the Lie group $G$ is an invertible mapping $\phi: G \rightarrow G$ such that $\phi(\boldsymbol{x}) \phi(\boldsymbol{y})=\phi(\boldsymbol{x y})$. If $L$ is a Lie algebra automorphism, then there exists a Lie group automorphism $\phi$ such that $L=\nabla \phi(\mathbf{0})$, and vice versa. Moreover,

$$
\phi\left(\mathrm{e}^{(\boldsymbol{\lambda})}\right)=\mathrm{e}^{(\nabla \boldsymbol{\phi}(0) \boldsymbol{\lambda})} .
$$

According to Thurston [18], if the subgroup $G_{\ell}$ of $G$ is to be discrete (that is, if the elements of $G_{\ell}$ are to be isolated, as points of $\mathbb{R}^{3}$ ) in the case that the generators $\mathrm{e}^{\left(\ell_{1}\right)}, \mathrm{e}^{\left(\ell_{2}\right)}, \mathrm{e}^{\left(\ell_{3}\right)}$ of $G_{\ell}$ are sufficiently small, then $G$ must be a nilpotent group and this implies, in this case, that the structure constants can be put in the form

$$
C_{k i j}=\varepsilon_{i j p} \lambda \nu_{p} \nu_{k}, \quad \lambda \in \mathbb{Q}, \quad \nu_{r} \in \mathbb{Z}, \quad r=1,2,3,
$$

where $\nu_{1}, \nu_{2}, \nu_{3}$ are relatively prime integers, Parry [12], Cermelli and Parry [5]. It follows that

$$
[\boldsymbol{x}, \boldsymbol{y}]=\lambda\left(\varepsilon_{k i j} x_{i} y_{j} \nu_{k}\right)\left(\nu_{r} \boldsymbol{e}_{r}\right), \quad[\boldsymbol{x},[\boldsymbol{y}, \boldsymbol{z}]]=0, \quad \boldsymbol{x}, \boldsymbol{y}, \boldsymbol{z} \in \mathbb{R}^{3} .
$$

We shall assume henceforward that the structure constants are such that (18) holds, for some choice of the rational number $\lambda$, some choice of the relatively prime integers $\nu_{1}, \nu_{2}, \nu_{3}$.

Now we recall the particular choice of the Lie group (equivalently, choice of composition function $\boldsymbol{\psi}$ ) that was made in [10], compatible with the given structure constants (equivalently, Lie bracket). Let $\boldsymbol{x}, \boldsymbol{y} \in \mathbb{R}^{3}$, then according to the Campbell Baker Hausdorff theorem, there exists a function $\boldsymbol{c}=\boldsymbol{c}(\boldsymbol{x}, \boldsymbol{y})$ such that

$$
\mathrm{e}^{(\boldsymbol{c})}=\mathrm{e}^{(\boldsymbol{x})} \mathrm{e}^{(\boldsymbol{y})} .
$$

It is a fact that the function $c$ has the properties required to be a Lie group composition function, and in the case that (18) holds we have $\boldsymbol{c} \equiv \boldsymbol{x}+\boldsymbol{y}+\frac{1}{2}[\boldsymbol{x}, \boldsymbol{y}]$. With Lie bracket given by (19), we choose

$$
\boldsymbol{\psi}(\boldsymbol{x}, \boldsymbol{y})=\boldsymbol{x}+\boldsymbol{y}+\frac{1}{2}[\boldsymbol{x}, \boldsymbol{y}]
$$

and call the corresponding $G$ the canonical Lie group $J$ associated with the Lie bracket (19), cf. Mal'cev [10]. This choice of composition function gives that; 
(i) the one-parameter subgroups of $J$ are straight lines through the origin,

(ii) $\mathrm{e}^{(\boldsymbol{x})} \equiv \exp (\boldsymbol{x})(\mathbf{0})=\boldsymbol{x}$,

(iii) from (17), $\phi(\boldsymbol{x})=\nabla \phi(\mathbf{0}) \boldsymbol{x}$.

According to the second of these facts, elements of the group $J$ may be identified with elements of the Lie algebra (here, $\mathbb{R}^{3}$ ). The third fact asserts that the automorphisms of $J$ can be written as linear transformations of $\mathbb{R}^{3}$ to itself. (From the point of view of continuum mechanics, this will allow us to interpret some symmetries of various structures as 'homogeneous elastic deformations').

2.2 The translation group $T_{\ell}$

The translation group $T_{\ell}$ of the discrete subgroup $G_{\ell} \subset J$ is defined by

$$
T_{\ell}=\left\{\boldsymbol{t} \in J ; \text { if } \boldsymbol{g} \in G_{\ell}, \boldsymbol{g}+\boldsymbol{t} \in G_{\ell}\right\} .
$$

Note that, since $\mathbf{0} \in G_{\ell}, T_{\ell} \subseteq G_{\ell}$. Let $\mathbb{Z}\left(G_{\ell}\right)$ be the centre of $G_{\ell}$, so $\mathbb{Z}\left(G_{\ell}\right):=\left\{\boldsymbol{x} \in G_{\ell}\right.$ : if $\left.\boldsymbol{y} \in G_{\ell},(\boldsymbol{x}, \boldsymbol{y})=\mathbf{0}\right\}$, where $(\boldsymbol{x}, \boldsymbol{y}):=\boldsymbol{x}^{-1} \boldsymbol{y}^{-1} \boldsymbol{x} \boldsymbol{y}, \boldsymbol{x}, \boldsymbol{y} \in G_{\ell}$. According to Cermelli and Parry [5]

$$
\boldsymbol{t} \in T_{\ell} \text { if and only if } \frac{1}{2}[\boldsymbol{t}, \boldsymbol{g}] \in \mathbb{Z}\left(G_{\ell}\right) \text { for all } \boldsymbol{g} \in G_{\ell} .
$$

It follows from this and Hall's identities [9] (see also (28) below) that $T_{\ell}$ is a normal subgroup of $G_{\ell}$. Also, $T_{\ell}$ is an additive subgroup of $\mathbb{R}^{3}$, and $\mathbb{Z}\left(G_{\ell}\right) \subseteq T_{\ell}$.

Proposition 1 If $\boldsymbol{g} \in G_{\ell}$, then $\boldsymbol{g}^{2}=2 \boldsymbol{g} \in T_{\ell}$.

Proof Let $G_{\ell}^{\prime}$ be the commutator subgroup of $G_{\ell}$, so $G_{\ell}^{\prime}$ is generated by all elements of the form $(\boldsymbol{x}, \boldsymbol{y}), \boldsymbol{x}, \boldsymbol{y} \in G_{\ell}$. By Hall's identities, if $\boldsymbol{g} \in G_{\ell}, \frac{1}{2}\left[\boldsymbol{g}^{2}, \boldsymbol{x}\right]=[\boldsymbol{g}, \boldsymbol{x}] \in G_{\ell}^{\prime} \subseteq$ $\mathbb{Z}\left(G_{\ell}\right)$ for all $\boldsymbol{x} \in G_{\ell}$. So the result follows by (23).

If $\boldsymbol{x}_{1}, \boldsymbol{x}_{2} \ldots \boldsymbol{x}_{p} \in \mathbb{R}^{3}, p$ an integer, let

$$
\left\langle\boldsymbol{x}_{1}, \boldsymbol{x}_{2}, \ldots \boldsymbol{x}_{p}\right\rangle:=\left\{\boldsymbol{x} \in \mathbb{R}^{3} ; \boldsymbol{x}=\sum_{i=1}^{p} n_{i} \boldsymbol{x}_{i}, n_{i} \in \mathbb{Z}, \quad i=1,2 \ldots p\right\},
$$

denote the integer linear span of the vectors $\boldsymbol{x}_{1}, \boldsymbol{x}_{2} \ldots \boldsymbol{x}_{p}$. Note that, since $T_{\ell}$ is a discrete additive subgroup of $\mathbb{R}^{3}$, it equals the integer linear span of a finite number of elements of the vector space $\mathbb{R}^{3}$, Bourbaki [3].

\section{Proposition 2}

(i) If $\boldsymbol{\alpha} \in G_{\ell}$, then $\boldsymbol{\alpha}+T_{\ell}=\boldsymbol{\alpha} T_{\ell}$.

(ii) Let $T_{\ell}=\left\langle\boldsymbol{x}_{1}, \boldsymbol{x}_{2} \ldots \boldsymbol{x}_{p}\right\rangle$ and suppose that one of $\boldsymbol{x}_{1}, \boldsymbol{x}_{2} \ldots \boldsymbol{x}_{p}$ is a generator of $\mathbb{Z}\left(G_{\ell}\right)$. Then each element of $T_{\ell}$ is expressible as a product of the elements $\boldsymbol{x}_{1}, \boldsymbol{x}_{2} \ldots$ $\boldsymbol{x}_{p} \in G_{\ell}$ and their inverses.

\section{Proof}

(i) $\boldsymbol{\alpha} T_{\ell} \subseteq \boldsymbol{\alpha}+T_{\ell}$ because, if $\boldsymbol{t} \in T_{\ell}$, then $\boldsymbol{\alpha} \boldsymbol{t}=\boldsymbol{\alpha}+\boldsymbol{t}+\frac{1}{2}[\boldsymbol{\alpha}, \boldsymbol{t}] \equiv \boldsymbol{\alpha}+\boldsymbol{s}$, where $\boldsymbol{s} \in T_{\ell}$ because $\boldsymbol{s}=\boldsymbol{t}+\frac{1}{2}[\boldsymbol{\alpha}, \boldsymbol{t}], \boldsymbol{t} \in T_{\ell}$ and $\frac{1}{2}[\boldsymbol{\alpha}, \boldsymbol{t}] \in \mathbb{Z}\left(G_{\ell}\right) \subseteq T_{\ell}$ by (23). The reverse inclusion is similar. 
(ii) Let $\boldsymbol{t}, \boldsymbol{t}^{\prime} \in T_{\ell}$. Since $\boldsymbol{t} \boldsymbol{t}^{\prime}=\boldsymbol{t}+\boldsymbol{t}^{\prime}+\frac{1}{2}\left[\boldsymbol{t}, \boldsymbol{t}^{\prime}\right]$ and $\frac{1}{2}\left[\boldsymbol{t}, \boldsymbol{t}^{\prime}\right] \in \mathbb{Z}\left(G_{\ell}\right)$ by (23), it follows that

$$
\boldsymbol{t}+\boldsymbol{t}^{\prime}=\boldsymbol{t} \boldsymbol{t}^{\prime}\left\{\frac{1}{2}\left[\boldsymbol{t}, \boldsymbol{t}^{\prime}\right]\right\}^{-1},
$$

and the result follows by induction.

Next we record some results from Cermelli and Parry [5], for later convenience. Recall that $\mathbb{Z}\left(G_{\ell}\right)$ is the centre of $G_{\ell}, G_{\ell}^{\prime}$ is the commutator subgroup. Then in the case in hand $G_{\ell}^{\prime} \subseteq \mathbb{Z}\left(G_{\ell}\right)$ and $\mathbb{Z}\left(G_{\ell}\right)$ has a single generator. The index of $G_{\ell}^{\prime}$ in $\mathbb{Z}\left(G_{\ell}\right)$ is denoted $k$ (so, if $s \in G_{\ell}$ generates $\mathbb{Z}\left(G_{\ell}\right)$, then $s^{k}$ generates $G_{\ell}^{\prime}$ ).

Theorem 3 Let $J$ be the Lie group with composition function (21), where $[\boldsymbol{x}, \boldsymbol{y}]$ is given by (19) with $\lambda=p / q \in \mathbb{Q}$, where $p, q \in \mathbb{Z}$ have no common factors, and $\nu_{1}, \nu_{2}, \nu_{3}$ are relatively prime. Define $\nu:=\nu_{1} \nu_{2} \nu_{3}$. Let $G_{\ell}$ be the discrete subgroup of $J$ generated by the three elements $\mathrm{e}^{\left(\ell_{1}\right)}, \mathrm{e}^{\left(\ell_{2}\right)}, \mathrm{e}^{\left(\ell_{3}\right)}\left(\right.$ so $\mathrm{e}^{\left(\boldsymbol{\ell}_{1}\right)}=\boldsymbol{\ell}_{1}(\mathbf{0})$, etc., by the remark following (16)). Then:

(i) $\mathbb{Z}\left(G_{\ell}\right)$ is generated by $\lambda \nu_{r} \ell_{r}(\mathbf{0}) / k=\lambda \boldsymbol{\nu} / k$ if we put $\boldsymbol{e}_{r} \equiv \boldsymbol{\ell}_{r}(\mathbf{0}), \boldsymbol{\nu} \equiv \nu_{r} \boldsymbol{e}_{r}$;

(ii) $G_{\ell}^{\prime}$ is generated by $\lambda \boldsymbol{\nu}$;

(iii) (a) $k=p$ if $\nu$ is even, or if ( $\nu$ is odd and $p \in 4 \mathbb{Z}$ ),

(b) $k=\frac{1}{2} p$ if $\nu$ is odd and $p \in 2 \mathbb{Z}, p \notin 4 \mathbb{Z}$,

(c) $k=2 p$ if $\nu$ is odd and $p \notin 2 \mathbb{Z}$;

(iv) If $k$ is even, $T_{\ell}=G_{\ell}$, and $T_{\ell}$ consists of (all) integer linear combinations of $\boldsymbol{e}_{1}, \boldsymbol{e}_{2}, \boldsymbol{e}_{3}, \lambda \nu / k$

(v) If $k$ is odd, $T_{\ell}$ consists of (all) integer linear combinations of $2 \boldsymbol{e}_{1}, 2 \boldsymbol{e}_{2}, 2 \boldsymbol{e}_{3}, \lambda \boldsymbol{\nu} / k$. $G_{\ell} / T_{\ell}$ has four elements which may be written as $T_{\ell}, \boldsymbol{\alpha} T_{\ell}, \boldsymbol{\beta} T_{\ell}, \boldsymbol{\alpha} \boldsymbol{\beta} T_{\ell}$, for some $\boldsymbol{\alpha}, \boldsymbol{\beta} \in G_{\ell}$.

Proof (i) and (ii) are Proposition 2, [5]. (iii) is Proposition 3, [5]. (iv) comes from remarks following (89), [5]. The first sentence of (v) is Proposition 6, [5]. Regarding the second sentence of (v), let $\boldsymbol{\alpha}=\boldsymbol{e}_{1}^{n_{1}} \boldsymbol{e}_{2}^{n_{2}} \boldsymbol{e}_{3}^{n_{3}}, \boldsymbol{\beta}=\boldsymbol{e}_{1}^{n_{1}^{\prime}} \boldsymbol{e}_{2}^{n_{2}^{\prime}} \boldsymbol{e}_{3}^{n_{3}^{\prime}}$, modulo $G_{\ell}^{\prime}$ (putting $\boldsymbol{e}^{\ell_{1}}=\boldsymbol{\ell}_{1}(\mathbf{0}) \equiv \boldsymbol{e}_{1}$, etc.). Then according to (98), [5], $\boldsymbol{\alpha}$ and $\boldsymbol{\beta}$ are equivalent modulo $T_{\ell}$ if and only if $n_{a}-n_{a}^{\prime}=\nu_{a} \bmod 2, a=1,2,3$. So if $T_{\ell}, \boldsymbol{\alpha} T_{\ell}$ and $\boldsymbol{\beta} T_{\ell}$ are distinct cosets in $G_{\ell}$, then each of $\left(n_{a}=\nu_{a} \bmod 2\right),\left(n_{a}^{\prime}=\nu_{a} \bmod 2\right),\left(n_{a}-n_{a}^{\prime}=\nu_{a} \bmod 2\right)$ is false for some choice of $a=1,2,3$. Since $\boldsymbol{\alpha} \boldsymbol{\beta}=\boldsymbol{e}_{1}^{n_{1}+n_{1}^{\prime}} \boldsymbol{e}_{2}^{n_{2}+n_{2}^{\prime}} \boldsymbol{e}_{3}^{n_{3}+n_{3}^{\prime}}$, modulo $G_{\ell}^{\prime}$, it follows that $\boldsymbol{\alpha} \boldsymbol{\beta} T_{\ell}$ is distinct from each of $T_{\ell}, \boldsymbol{\alpha} T_{\ell}, \boldsymbol{\beta} T_{\ell}$ (since, for example, it is false that $n_{a}+n_{a}^{\prime}=\nu_{a} \bmod 2, a=1,2,3$, so $\boldsymbol{\alpha} \boldsymbol{\beta} T_{\ell} \neq T_{\ell}$ ). It is shown in [5] that $G_{\ell} / T_{\ell}$ has four elements.

\subsection{Canonical coordinates for $G_{\ell}$}

$G_{\ell}$ is generated by three elements $\mathrm{e}^{\left(\ell_{1}\right)}, \mathrm{e}^{\left(\ell_{2}\right)}, \mathrm{e}^{\left(\ell_{3}\right)}$. Thus if $\boldsymbol{g} \in G_{\ell}, \boldsymbol{g}$ is expressible as a product of the three generators and their inverses. According to Mal'cev [10], in a nilpotent group (in particular, in a three dimensional nilpotent group, where the structure constants have the form (18)) one may choose the three generators in such a way that the Lie bracket has a particular simple form: there are generators of $G_{\ell} \subset J$ and corresponding Lie algebra elements $\boldsymbol{c}_{1}, \boldsymbol{c}_{2}, \boldsymbol{c}_{3} \in \mathbb{R}^{3}$ such that for some integer $k$,

$$
\left[\boldsymbol{c}_{1}, \boldsymbol{c}_{2}\right]=k \boldsymbol{c}_{3},\left[\boldsymbol{c}_{1}, \boldsymbol{c}_{3}\right]=\left[\boldsymbol{c}_{2}, \boldsymbol{c}_{3}\right]=\mathbf{0}, \quad k \in \mathbb{Z} .
$$


Recalling that group and algebra elements may be identified in $J$, Mal'cev shows further that any $\boldsymbol{g} \in G_{\ell} \subset J$ may be written in the form

$$
\boldsymbol{g}=\boldsymbol{c}_{1}^{\alpha} \boldsymbol{c}_{2}^{\beta} \boldsymbol{c}_{3}^{\gamma}, \quad \alpha, \beta, \gamma \in \mathbb{Z}
$$

(Note that this relation (27) is a generalization of the expression of a point $\boldsymbol{x}$ of a perfect lattice with basis $\boldsymbol{e}_{1}, \boldsymbol{e}_{2}, \boldsymbol{e}_{3}$ as $\left.\boldsymbol{x}=\alpha \boldsymbol{e}_{1}+\beta \boldsymbol{e}_{2}+\gamma \boldsymbol{e}_{3}, \alpha, \beta, \gamma \in \mathbb{Z}\right)$.

Let $G_{\ell}^{\prime}$ be the commutator subgroup of $G_{\ell}$ and recall the following identities, due to Hall [9],

$$
(\boldsymbol{x}, \boldsymbol{y} \boldsymbol{z})=(\boldsymbol{x}, \boldsymbol{z})(\boldsymbol{x}, \boldsymbol{y})((\boldsymbol{x}, \boldsymbol{y}), \boldsymbol{z}), \quad(\boldsymbol{x} \boldsymbol{y}, \boldsymbol{z})=(\boldsymbol{x}, \boldsymbol{z})((\boldsymbol{x}, \boldsymbol{z}), \boldsymbol{y})(\boldsymbol{y}, \boldsymbol{z}) .
$$

In the nilpotent case, one has $((\boldsymbol{x}, \boldsymbol{y}), \boldsymbol{z})=\mathbf{0}$ for all $\boldsymbol{x}, \boldsymbol{y}, \boldsymbol{z} \in G_{\ell}$, so that $(\boldsymbol{x}, \boldsymbol{y} \boldsymbol{z})=$ $(\boldsymbol{x}, \boldsymbol{z})(\boldsymbol{x}, \boldsymbol{y}),(\boldsymbol{x} \boldsymbol{y}, \boldsymbol{z})=(\boldsymbol{x}, \boldsymbol{z})(\boldsymbol{y}, \boldsymbol{z})$. It follows that if $\boldsymbol{x}=\boldsymbol{c}_{1}^{n_{1}} \boldsymbol{c}_{2}^{n_{2}} \boldsymbol{c}_{3}^{n_{3}}, \boldsymbol{y}=\boldsymbol{c}_{1}^{m_{1}} \boldsymbol{c}_{2}^{m_{2}} \boldsymbol{c}_{3}^{m_{3}}$ for integers $n_{i}, m_{i}, i=1,2,3$, then $(\boldsymbol{x}, \boldsymbol{y})$ is a product of terms of the form $\left(\boldsymbol{c}_{i}, \boldsymbol{c}_{j}\right)$. Also, note that, for $\boldsymbol{x}, \boldsymbol{y} \in J$, with $\boldsymbol{x}^{-1}=-\boldsymbol{x}$, etc.,

$$
(\boldsymbol{x}, \boldsymbol{y})=\left(-\boldsymbol{x}-\boldsymbol{y}+\frac{1}{2}[\boldsymbol{x}, \boldsymbol{y}]\right)\left(\boldsymbol{x}+\boldsymbol{y}+\frac{1}{2}[\boldsymbol{x}, \boldsymbol{y}]\right)=[\boldsymbol{x}, \boldsymbol{y}],
$$

using properties of $[\cdot, \cdot]$ given above. In particular, (26) may be rewritten as

$$
\left(c_{1}, c_{2}\right)=c_{3}^{k}, \quad\left(c_{1}, c_{3}\right)=\left(c_{2}, c_{3}\right)=\mathbf{0},
$$

and it follows that $G_{\ell}^{\prime}$ is generated by $c_{3}^{k}$.

Also, let $\mathbb{Z}\left(G_{\ell}\right)$ be the centre of $G_{\ell}$. If $\boldsymbol{x}=\boldsymbol{c}_{1}^{\alpha} \boldsymbol{c}_{2}^{\beta} \boldsymbol{c}_{3}^{\gamma}$, then $\left(\boldsymbol{x}, \boldsymbol{c}_{1}\right)=\left(\boldsymbol{c}_{2}^{\beta}, \boldsymbol{c}_{1}\right)=$ $\left(\boldsymbol{c}_{2}, \boldsymbol{c}_{1}\right)^{\beta}=\boldsymbol{c}_{3}^{-\beta k}$, and $\left(\boldsymbol{x}_{1}, \boldsymbol{c}_{2}\right)=\boldsymbol{c}_{3}^{\alpha k}$, so if $\boldsymbol{x} \in \mathbb{Z}\left(G_{\ell}\right)$, then $\boldsymbol{x}=\boldsymbol{c}_{3}^{\gamma}$ for some integer $\gamma$. Moreover $\left(\boldsymbol{c}_{3}^{\gamma}, \boldsymbol{y}\right)=\mathbf{0}$ for all $\gamma \in \mathbb{Z}$, so $\mathbb{Z}\left(G_{\ell}\right)$ is generated by $\boldsymbol{c}_{3}$ (this is consistent with Theorem 3).

The integer $k$ is the index of the (normal) subgroup $G_{\ell}^{\prime}$ in $\mathbb{Z}\left(G_{\ell}\right)$, and this integer will figure prominently in what follows. Note also that Mal'cev [10] shows that every automorphism of $G_{\ell}$ extends uniquely to an automorphism of $J$. Given fact (iii) at the end of section 2.1, this implies that the automorphisms of $G_{\ell} \subset J$ are (restrictions of) linear transformations of $\mathbb{R}^{3}$ to itself.

\section{Generators of $G_{c}$}

Here we presume that a canonical basis $\boldsymbol{c}_{1}, \boldsymbol{c}_{2}, \boldsymbol{c}_{3}$ of a discrete subgroup $G_{\boldsymbol{c}} \subset J$ is given, and find necessary and sufficient conditions that elements $\boldsymbol{e}_{1}, \boldsymbol{e}_{2}, \boldsymbol{e}_{3} \in G_{\boldsymbol{c}}$ also generate $G_{\boldsymbol{c}}$.

\subsection{Canonical basis}

Let $J=\left(\mathbb{R}^{3}, \cdot\right)$ be the canonical nilpotent Lie group with composition function (multiplication of group elements) defined by

$$
\boldsymbol{x} \boldsymbol{y}=\boldsymbol{x}+\boldsymbol{y}+\frac{1}{2}[\boldsymbol{x}, \boldsymbol{y}], \quad \boldsymbol{x}, \boldsymbol{y} \in \mathbb{R}^{3} .
$$


Now let $\boldsymbol{c}_{1}, \boldsymbol{c}_{2}, \boldsymbol{c}_{3}$ be a canonical basis of some discrete group $G_{\boldsymbol{c}} \subset J$. Then there exists an integer $k_{c}$, which is the index of $G_{\boldsymbol{c}}^{\prime} \equiv\left[G_{\boldsymbol{c}}, G_{\boldsymbol{c}}\right]$ in $\mathbb{Z}\left(G_{\boldsymbol{c}}\right)$, with the property that

$$
\left[\boldsymbol{c}_{1}, \boldsymbol{c}_{2}\right]=k_{c} \boldsymbol{c}_{3}, \quad\left[\boldsymbol{c}_{1}, \boldsymbol{c}_{3}\right]=\left[\boldsymbol{c}_{2}, \boldsymbol{c}_{3}\right]=0 .
$$

With respect to that particular basis, writing $\boldsymbol{x}=x_{i} \boldsymbol{c}_{i}, \boldsymbol{y}=y_{i} \boldsymbol{c}_{i}$, one has

$$
[\boldsymbol{x}, \boldsymbol{y}]=x_{i} y_{j} \varepsilon_{i j p} k_{c} \delta_{p 3}\left(\delta_{r 3} \boldsymbol{c}_{r}\right)
$$

a particular form of equation (19) above.

Let $T_{\boldsymbol{c}}$ be the translational group corresponding to $G_{\boldsymbol{c}}$. According to remarks in section $2.3, G_{\boldsymbol{c}}^{\prime}$ is generated by $\boldsymbol{c}_{3}^{k_{c}}$, the index of $G_{\boldsymbol{c}}^{\prime}$ in $\mathbb{Z}\left(G_{\boldsymbol{c}}\right)$ is $k_{c}$, and $\mathbb{Z}\left(G_{\boldsymbol{c}}\right)$ is generated by $\boldsymbol{c}_{3}$. (These facts are evident from (32), directly). From Cermelli and Parry [5], or Theorem 3 above, we have that

$$
\begin{aligned}
& T_{\boldsymbol{c}}=\left\langle\boldsymbol{c}_{1}, \boldsymbol{c}_{2}, \boldsymbol{c}_{3}\right\rangle, \quad k_{c} \text { even } \\
& =\left\langle 2 c_{1}, 2 c_{2}, c_{3}\right\rangle, \quad k_{c} \text { odd } .
\end{aligned}
$$

3.2 Conditions necessary and sufficient that $e_{1}, e_{2}, e_{3} \in G_{c}$ generate $G_{c}$

In this section we find the set of all generators of the given discrete group $G_{\boldsymbol{c}}$. Let $\boldsymbol{e}_{1}, \boldsymbol{e}_{2}, \boldsymbol{e}_{3} \in G_{\boldsymbol{c}}$ and consider to begin with the subgroup of $G_{\boldsymbol{c}}$ which consists of all products of the elements $\boldsymbol{e}_{1}, \boldsymbol{e}_{2}, \boldsymbol{e}_{3}$ and their inverses, denoted $G_{\boldsymbol{e}}$. We shall assume that $\boldsymbol{e}_{1}, \boldsymbol{e}_{2}, \boldsymbol{e}_{3}$ provide a basis of $\mathbb{R}^{3}$.

Proposition 4 With respect to the basis vectors $\boldsymbol{e}_{1}, \boldsymbol{e}_{2}, \boldsymbol{e}_{3}$, the composition function $\boldsymbol{x} \boldsymbol{y}=\boldsymbol{x}+\boldsymbol{y}+\frac{1}{2}[\boldsymbol{x}, \boldsymbol{y}]$ with Lie bracket given by (33) has the form

$$
\boldsymbol{x} \boldsymbol{y}=\boldsymbol{x}+\boldsymbol{y}+\frac{\theta k_{c}}{2 \Gamma} \varepsilon_{i j p} x_{i} y_{j} \nu_{p}\left(\nu_{r} \boldsymbol{e}_{r}\right)
$$

for some integers $\Gamma, \nu_{i}, i=1,2,3$, where $\theta=1$ if $k_{c}$ is even and $\theta=2$ if $k_{c}$ is odd. Also $\left[\boldsymbol{e}_{i}, \boldsymbol{e}_{j}\right]=\frac{\theta k_{c}}{\Gamma} \varepsilon_{i j p} \nu_{p}\left(\nu_{r} \boldsymbol{e}_{r}\right)$.

\section{Proof}

(i) Suppose that $k_{c}$ is even. Then according to Cermelli and Parry [5], $G_{\boldsymbol{c}}=T_{\boldsymbol{c}}=$ $\left\langle\boldsymbol{c}_{1}, \boldsymbol{c}_{2}, \boldsymbol{c}_{3}\right\rangle$. Since $\boldsymbol{e}_{i} \in G_{\boldsymbol{c}}, i=1,2,3$, there exists a matrix $\gamma \equiv\left(\gamma_{i j}\right)$ of integers, with determinant $\Gamma:=\operatorname{det}(\gamma)$ such that

$$
\boldsymbol{e}_{i}=\gamma_{i j} \boldsymbol{c}_{j}
$$

So $\left[\boldsymbol{e}_{i}, \boldsymbol{e}_{j}\right]=\gamma_{i p} \gamma_{j q}\left[\boldsymbol{c}_{p}, \boldsymbol{c}_{q}\right]=\varepsilon_{p q 3} \gamma_{i p} \gamma_{j q} k_{c} \boldsymbol{c}_{3}$. Define, and observe that,

$$
p_{\ell k}:= \pm \frac{1}{2} \varepsilon_{p q \ell} \varepsilon_{i j k} \gamma_{i p} \gamma_{j q}, \quad p_{\ell k} \in \mathbb{Z}, \quad \ell, k=1,2,3 .
$$

Then

$$
p_{\ell k} \gamma_{k m}= \pm \frac{1}{2} \varepsilon_{p q \ell} \varepsilon_{p q m} \Gamma= \pm \Gamma \delta_{\ell m}, \quad \gamma^{-1}= \pm\left(\frac{p_{\ell k}}{\Gamma}\right)
$$

and so

$$
\boldsymbol{c}_{j}= \pm \frac{1}{\Gamma} p_{j i} \boldsymbol{e}_{i}, \quad \Gamma, p_{i j} \in \mathbb{Z}, \quad i, j=1,2,3 .
$$


Thus,

$$
\begin{aligned}
{\left[\boldsymbol{e}_{1}, \boldsymbol{e}_{2}\right] } & =\frac{1}{2} \varepsilon_{i j 3}\left[\boldsymbol{e}_{i}, \boldsymbol{e}_{j}\right]=\frac{1}{2} \varepsilon_{i j 3} \varepsilon_{p q 3} \gamma_{i p} \gamma_{j q} k_{c} \boldsymbol{c}_{3} \\
& = \pm p_{33} k_{c} \boldsymbol{c}_{3}=p_{33} \frac{k_{c}}{\Gamma}\left(p_{3 i} \boldsymbol{e}_{i}\right) .
\end{aligned}
$$

Similarly one finds $\left[\boldsymbol{e}_{2}, \boldsymbol{e}_{3}\right]= \pm p_{31} k_{c} \boldsymbol{c}_{3},\left[\boldsymbol{e}_{3}, \boldsymbol{e}_{1}\right]= \pm p_{32} k_{c} \boldsymbol{c}_{3}$.

Define $\nu_{i}:=p_{3 i}$, so $\nu_{i} \in \mathbb{Z}, i=1,2,3$. Then

$$
[\boldsymbol{x}, \boldsymbol{y}]=x_{i} y_{j}\left[\boldsymbol{e}_{i}, \boldsymbol{e}_{j}\right]=x_{i} y_{j} \varepsilon_{i j p} \nu_{p} \frac{k_{c}}{\Gamma}\left(\nu_{r} \boldsymbol{e}_{r}\right),
$$

noting that $\left[\boldsymbol{e}_{i}, \boldsymbol{e}_{j}\right]=\varepsilon_{i j p} \nu_{p} \frac{k_{c}}{T}\left(\nu_{r} \boldsymbol{e}_{r}\right)$. This proves the result in the case that $k_{c}$ is even.

(ii) Suppose that $k_{c}$ is odd. From Cermelli and Parry [5], $T_{\boldsymbol{c}}=\left\langle 2 \boldsymbol{c}_{1}, 2 \boldsymbol{c}_{2}, \boldsymbol{c}_{3}\right\rangle$ and $T_{\boldsymbol{c}}$ is a normal subgroup of $G_{\boldsymbol{c}}$. Note that if $\boldsymbol{g} \in G_{\boldsymbol{c}}$, then $\boldsymbol{g}^{2} \in T_{\boldsymbol{c}}$, from proposition 1 in section 2.2. Hence, since $\boldsymbol{e}_{i} \in G_{\boldsymbol{c}}, i=1,2,3$, there exists a matrix $\gamma=\left(\gamma_{i j}\right)$ of integers, with determinant $\Gamma:=\operatorname{det}(\gamma) \in \mathbb{Z}$, such that

$$
2 \boldsymbol{e}_{i}=\gamma_{i j} \boldsymbol{c}_{j}^{\prime}
$$

where one defines $\boldsymbol{c}_{1}^{\prime}=2 \boldsymbol{c}_{1}, \boldsymbol{c}_{2}^{\prime}=2 \boldsymbol{c}_{2}, \boldsymbol{c}_{3}^{\prime}=\boldsymbol{c}_{3}$. Note that $\left[\boldsymbol{c}_{1}^{\prime}, \boldsymbol{c}_{2}^{\prime}\right]=4 k_{c} \boldsymbol{c}_{3}$, so $\left[\boldsymbol{c}_{p}^{\prime}, \boldsymbol{c}_{q}^{\prime}\right]=4 k_{c} \varepsilon_{p q 3} \boldsymbol{c}_{3}$. So from (42)

$$
\left[\boldsymbol{e}_{i}, \boldsymbol{e}_{j}\right]=\frac{1}{4} \gamma_{i p} \gamma_{j q}\left[\boldsymbol{c}_{p}^{\prime}, \boldsymbol{c}_{q}^{\prime}\right]=\varepsilon_{p q 3} \gamma_{i p} \gamma_{j q} k_{c} \boldsymbol{c}_{3},
$$

which has the form shown above (37). Define $p_{\ell k}$ as in (37) above and note that, correspondingly, $\gamma^{-1}= \pm \frac{1}{\Gamma} p$. Hence $\boldsymbol{c}_{1}^{\prime}=2 \boldsymbol{c}_{1}=\gamma_{1 j}^{-1}\left(2 \boldsymbol{e}_{j}\right)= \pm \frac{2}{\Gamma} p_{1 j} \boldsymbol{e}_{j}$, etc.. Thus

$$
c_{1}= \pm \frac{p_{1 j} \boldsymbol{e}_{j}}{\Gamma}, \quad \boldsymbol{c}_{2}= \pm \frac{p_{2 j} \boldsymbol{e}_{j}}{\Gamma}, \quad \boldsymbol{c}_{3}= \pm \frac{2 p_{3 j} \boldsymbol{e}_{j}}{\Gamma}
$$

Let $\nu_{i}:=p_{3 i}$ as before, then we have

$$
\left[\boldsymbol{e}_{1}, \boldsymbol{e}_{2}\right]= \pm \nu_{3} k_{c} \boldsymbol{c}_{3}, \quad\left[\boldsymbol{e}_{2}, \boldsymbol{e}_{3}\right]= \pm \nu_{1} k_{c} \boldsymbol{c}_{3}, \quad\left[\boldsymbol{e}_{3}, \boldsymbol{e}_{1}\right]= \pm \nu_{2} k_{c} \boldsymbol{c}_{3},
$$

from which the second assertion in the Proposition follows, in this case, using $(44)_{3}$. The form of the composition function follows from the expression for $\left[\boldsymbol{e}_{i}, \boldsymbol{e}_{j}\right]$.

Now since $\boldsymbol{x} \boldsymbol{y}$ is expressed in terms of the basis $\boldsymbol{e}_{1}, \boldsymbol{e}_{2}, \boldsymbol{e}_{3}$ in proposition 4 , Theorem 3 allows us to calculate $G_{\boldsymbol{e}}^{\prime}, \mathbb{Z}\left(G_{\boldsymbol{e}}\right)$, and the index of $G_{\boldsymbol{e}}^{\prime}$ in $\mathbb{Z}\left(G_{\boldsymbol{e}}\right)$, denoted $k_{\boldsymbol{e}}$. First we have:

Proposition $5 G_{\boldsymbol{e}}^{\prime}=G_{\boldsymbol{c}}^{\prime}$ if and only if the integers $\nu_{1}, \nu_{2}, \nu_{3}$ are relatively prime. In that case, $G_{\boldsymbol{e}}^{\prime}$ is generated by $k_{c} \boldsymbol{c}_{3}$ (as is $G_{\boldsymbol{c}}^{\prime}$ ).

Proof Let $\nu_{i}=d \nu_{i}^{\prime}, i=1,2,3$, where $d:=h c f\left(\nu_{1}, \nu_{2}, \nu_{3}\right)$, the highest common factor of $\nu_{1}, \nu_{2}, \nu_{3}$. Then the expression for $\boldsymbol{x} \boldsymbol{y}$, with respect to the basis $\boldsymbol{e}_{1}, \boldsymbol{e}_{2}, \boldsymbol{e}_{3}$, is

$$
\boldsymbol{x} \boldsymbol{y}=\boldsymbol{x}+\boldsymbol{y}+\frac{\theta d^{2}}{2 \Gamma} \varepsilon_{i j p} x_{i} y_{j} \nu_{p}^{\prime} k_{c}\left(\nu_{r}^{\prime} \boldsymbol{e}_{r}\right)
$$

It follows that $G_{\boldsymbol{e}}^{\prime}$ is generated by $\frac{\theta d^{2} k_{c}}{\Gamma}\left(\nu_{r}^{\prime} \boldsymbol{e}_{r}\right)=\frac{\theta d k_{c}}{\Gamma} \nu_{r} \boldsymbol{e}_{r}=d k_{c} \boldsymbol{c}_{3}$ (as is evident from (45)). But $G_{\boldsymbol{c}}^{\prime}$ is generated by $k_{c} \boldsymbol{c}_{3}$. Hence $d= \pm 1$ and the integers $\nu_{1}, \nu_{2}, \nu_{3}$ are relatively prime, in case $G_{\boldsymbol{e}}^{\prime}=G_{\boldsymbol{c}}^{\prime}$. 
Proposition $6 G_{\boldsymbol{e}}^{\prime}=G_{\boldsymbol{c}}^{\prime}$ and $\mathbb{Z}\left(G_{\boldsymbol{e}}\right)=\mathbb{Z}\left(G_{\boldsymbol{c}}\right)$ if and only if the integers $\nu_{1}, \nu_{2}, \nu_{3}$ are relatively prime, and also (with $\nu:=\nu_{1} \nu_{2} \nu_{3}$ and with $\Gamma:=\operatorname{det}(\gamma)$, and $\gamma=\left(\gamma_{i j}\right)$ such that (36) or (42) holds),

(i) if $k_{c} \in 4 \mathbb{Z}, h c f\left(k_{c}, \Gamma\right)=1$,

(ii) if $k_{c} \in 2 \mathbb{Z}, k_{c} \notin 4 \mathbb{Z}$, either ( $\nu$ is even and $h c f\left(k_{c}, \Gamma\right)=1$ or ( $\nu$ is odd and $\left.h c f\left(k_{c}, \Gamma\right)=2\right)$,

(iii) if $k_{c} \notin 2 \mathbb{Z}, h c f\left(k_{c}, \Gamma\right)=1$ and either $(\nu, \Gamma$ are both even) or ( $\nu, \Gamma$ are both odd).

Proof Let $k_{e}$ be the index of $G_{\boldsymbol{e}}^{\prime}$ in $\mathbb{Z}\left(G_{\boldsymbol{e}}\right)$. One only has to find the conditions that $k_{c}=k_{e}$, which may be found from Theorem 3 .

Suppose that $k_{c}$ is even, so $\theta=1$ in (35). Comparing (35) with (19) we see that in this case we have $\lambda=p / q=k / \Gamma$ where $h c f(p, q)=1$ and so $p=k / d$ where $d:=h c f\left(k_{c}, \Gamma\right)$. Then by Theorem 3 :

(ai) $k_{e}=k_{c} / d$ if $\nu$ is even,

(aii) $k_{e}=k_{c} / d$ if $\nu$ is odd and $k_{c} / d \in 4 \mathbb{Z}$,

(aiii) $k_{e}=k_{c} / 2 d$ if $\nu$ is odd and $k_{c} / d \in 2 \mathbb{Z}, k_{c} / d \notin 4 \mathbb{Z}$, (this implies $k_{e}$ is odd).

(aiv) $k_{e}=2 k_{c} / d$ if $\nu$ is odd and $k_{c} / d \notin 2 \mathbb{Z}$.

From these observations, it follows that in the case where $k_{c}$ is even, $k_{c}=k_{e}$ if and only if

(bi) if $\nu$ is even, $h c f\left(k_{c}, \Gamma\right)=1$,

(bii) if $\nu$ is odd, $h c f\left(k_{c}, \Gamma\right)=1, k_{c} \in 4 \mathbb{Z}$,

(biii) if $\nu$ is odd, $h c f\left(k_{c}, \Gamma\right)=2, k_{c} / 2 \notin 2 \mathbb{Z}$,

noting that $k_{c} \neq k_{e}$ in case (aiii). Then, (bi) and (bii) give (i) in the proposition, (bi) and (biii) give (ii) in the proposition.

Next, if $k_{c}$ is odd, so $\theta=2$ in (35), we have from Theorem 3 , again with $d:=$ $h c f\left(k_{c}, \Gamma\right)$ (noting that if $2 k_{c} / \Gamma=p / q$ and $p, q \in \mathbb{Z}$ have no common factors, then $p=k_{c} / d, q=\Gamma / 2 d$ if $\Gamma$ is even, and $p=2 k_{c} / d, q=\Gamma / d$ if $\Gamma$ is odd):

(ci) if $\nu$ is even, $\Gamma$ is even, $k_{e}=k_{c} / d$,

(cii) if $\nu$ is even, $\Gamma$ is odd, $k_{e}=2 k_{c} / d$ (this implies $k_{e}$ is even),

(ciii) if $\nu$ is odd, $\Gamma$ is even, $k_{c} / d \in 4 \mathbb{Z}, k_{e}=k_{c} / d$ (this implies $k_{e} \in 4 \mathbb{Z}$ ),

(civ) if $\nu$ is odd, $\Gamma$ is odd, $2 k_{c} / d \in 4 \mathbb{Z}, k_{e}=2 k_{c} / d$ (this implies $k_{e} \in 4 \mathbb{Z}$ ),

(cv) if $\nu$ is odd, $\Gamma$ is even, $k_{c} / d \in 2 \mathbb{Z}, k_{c} / d \notin 4 \mathbb{Z}, k_{e}=k_{c} / 2 d$ (this implies $k_{e}<k_{c}$ ),

(cvi) if $\nu$ is odd, $\Gamma$ is odd, $2 k_{c} / d \in 2 \mathbb{Z}, 2 k_{c} / d \notin 4 \mathbb{Z}$, then $k_{e}=k_{c} / d$,

(cvii) if $\nu$ is odd, $\Gamma$ is even, $k_{c} / d \notin 2 \mathbb{Z}, k_{e}=2 k_{c} / d$, (this implies $k_{e}$ is even),

(cviii) if $\nu$ is odd, $\Gamma$ is odd, $2 k_{c} / d \notin 2 \mathbb{Z}, k_{e}=4 k_{c} / d$ (this case is empty).

Only cases (ci) and (cvi) survive, if $k_{e}=k_{c} \notin 2 \mathbb{Z}$, and they give statement (iii) of the proposition.

Theorem $7 G_{\boldsymbol{c}}=G_{\boldsymbol{e}}$ if and only if there exist integers $l_{0}, m_{0}, \Gamma$, a matrix $A \in$ $G L_{3}(\mathbb{Z})$ with third row $\nu_{1}, \nu_{2}, \nu_{3}$, such that the conditions of proposition 6 are satisfied, and

(i) if $k_{c}$ is even,

$$
\left(\begin{array}{ccc}
1 & 0 & -l_{0} \\
0 & 1 & -m_{0} \\
0 & 0 & \pm \Gamma
\end{array}\right)\left(\begin{array}{l}
c_{1} \\
c_{2} \\
c_{3}
\end{array}\right)=A\left(\begin{array}{l}
e_{1} \\
e_{2} \\
e_{3}
\end{array}\right)
$$


(ii) if $k_{c}$ is odd,

$$
\left(\begin{array}{ccc}
1 & 0 & -\left(2 l_{0}+\theta(\boldsymbol{l})\right) \\
0 & 1 & -\left(2 m_{0}+\theta(\boldsymbol{m})\right) \\
0 & 0 & \pm \Gamma
\end{array}\right)\left(\begin{array}{c}
\boldsymbol{c}_{1} \\
\boldsymbol{c}_{2} \\
\frac{1}{2} \boldsymbol{c}_{3}
\end{array}\right)=A\left(\begin{array}{c}
\boldsymbol{e}_{1} \\
\boldsymbol{e}_{2} \\
\boldsymbol{e}_{3}
\end{array}\right)
$$

where

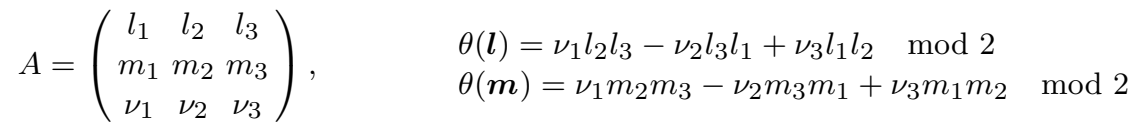

and $\Gamma \operatorname{det}\left\{\boldsymbol{c}_{i}\right\}=\operatorname{det}\left\{\boldsymbol{e}_{i}\right\}$.

\section{Proof}

(i) Suppose that $G_{\boldsymbol{c}}=G_{\boldsymbol{e}}$ and $k_{c}=k_{e}$ is even. Then the conditions of proposition 6 hold and also $T_{\boldsymbol{c}}=T_{\boldsymbol{e}}, T_{\boldsymbol{c}}=G_{\boldsymbol{c}}$ regarding $T_{\boldsymbol{c}}$ as a (normal) subgroup of $G_{\boldsymbol{c}}$. Thus

$$
\left\langle\boldsymbol{c}_{1}, \boldsymbol{c}_{2}, \boldsymbol{c}_{3}\right\rangle=\left\langle\boldsymbol{e}_{1}, \boldsymbol{e}_{2}, \boldsymbol{e}_{3}, \frac{\nu_{i} \boldsymbol{e}_{i}}{\Gamma}\right\rangle,
$$

with $\boldsymbol{c}_{3}= \pm \nu_{i} \boldsymbol{e}_{i} / \Gamma$. Hence there exist integers $l_{0}, l_{1}, l_{2}, l_{3}, m_{0}, m_{1}, m_{2}, m_{3}$ such that

$$
\begin{aligned}
\boldsymbol{c}_{1} & =\ell_{1} \boldsymbol{e}_{1}+\ell_{2} \boldsymbol{e}_{2}+\ell_{3} \boldsymbol{e}_{3}+\ell_{0} \frac{\nu_{i} \boldsymbol{e}_{i}}{\Gamma}, \\
\boldsymbol{c}_{2} & =m_{1} \boldsymbol{e}_{1}+m_{2} \boldsymbol{e}_{2}+m_{3} \boldsymbol{e}_{3}+m_{0} \frac{\nu_{i} \boldsymbol{e}_{i}}{\Gamma}, \\
\pm \Gamma \boldsymbol{c}_{3} & =\nu_{1} \boldsymbol{e}_{1}+\nu_{2} \boldsymbol{e}_{2}+\nu_{3} \boldsymbol{e}_{3} .
\end{aligned}
$$

If $A$ is the matrix with rows $\left(l_{1}, l_{2}, l_{3}\right),\left(m_{1}, m_{2}, m_{3}\right),\left(\nu_{1}, \nu_{2}, \nu_{3}\right)$, it follows that (47) holds, with $A$ an integral matrix, but as yet no information regarding $\operatorname{det} A$ is known. Taking the determinant of (47), $\pm \Gamma \boldsymbol{c}_{1} \wedge \boldsymbol{c}_{2} \cdot \boldsymbol{c}_{3}=\operatorname{det} A \boldsymbol{e}_{1} \wedge \boldsymbol{e}_{2} \cdot \boldsymbol{e}_{3}$. From $\boldsymbol{e}_{i}=\gamma_{i j} \boldsymbol{c}_{j}$, we have $\operatorname{det}\left\{\boldsymbol{e}_{i}\right\}=\Gamma \operatorname{det}\left\{\boldsymbol{c}_{i}\right\}$, and comparing with (47) we get $A \in G L_{3}(\mathbb{Z})$.

Conversely if (47) holds with $A \in G L_{3}(\mathbb{Z})$, then $\boldsymbol{e}_{i}=\gamma_{i j} \boldsymbol{c}_{j}$ where

$$
\gamma=A^{-1}\left(\begin{array}{ccc}
1 & 0 & -l_{0} \\
0 & 1 & -m_{0} \\
0 & 0 & \pm \Gamma
\end{array}\right)
$$

Also $\operatorname{det} \gamma=\Gamma\left(\right.$ since $\left.\Gamma \operatorname{det}\left\{\boldsymbol{c}_{i}\right\}=\operatorname{det}\left\{\boldsymbol{e}_{i}\right\}\right)$. Since

$$
\boldsymbol{c}_{1}-l_{0} \boldsymbol{c}_{3}=l_{i} \boldsymbol{e}_{i}, \quad \boldsymbol{c}_{2}-m_{0} \boldsymbol{c}_{3}=m_{i} \boldsymbol{e}_{i}, \quad \pm \Gamma \boldsymbol{c}_{3}=\nu_{i} \boldsymbol{e}_{i},
$$

we have

$$
T_{\boldsymbol{e}}=\left\langle\boldsymbol{e}_{1}, \boldsymbol{e}_{2}, \boldsymbol{e}_{3}, \frac{\nu_{i} \boldsymbol{e}_{i}}{\Gamma}\right\rangle=\left\langle\boldsymbol{l}, \boldsymbol{m}, \boldsymbol{\nu}, \frac{\boldsymbol{\nu}}{\Gamma}\right\rangle=\left\langle\boldsymbol{l}, \boldsymbol{m}, \frac{\boldsymbol{\nu}}{\Gamma}\right\rangle=\left\langle\boldsymbol{c}_{1}, \boldsymbol{c}_{2}, \boldsymbol{c}_{3}\right\rangle=T_{\boldsymbol{c}},
$$

(writing $\boldsymbol{l}=l_{i} \boldsymbol{e}_{i}$, etc.).

The conditions of proposition 6 ensure that $k_{c}=k_{e}$, so that $\boldsymbol{c}_{3}= \pm \nu_{i} \boldsymbol{e}_{i} / \Gamma$ is a generator of $\mathbb{Z}\left(G_{\boldsymbol{e}}\right)$. Hence by proposition $2, \boldsymbol{e}_{1}, \boldsymbol{e}_{2}, \boldsymbol{e}_{3}$ generate $T_{\boldsymbol{e}}=G_{\boldsymbol{e}}$ (multiplicatively), since $\nu_{i} \boldsymbol{e}_{i} / \Gamma \in \mathbb{Z}\left(G_{\boldsymbol{e}}\right)$ implies that $\nu_{i} \boldsymbol{e}_{i} / \Gamma$ is a product of $\boldsymbol{e}_{1}, \boldsymbol{e}_{2}, \boldsymbol{e}_{3}$ and their inverses. Hence, $\boldsymbol{e}_{1}, \boldsymbol{e}_{2}, \boldsymbol{e}_{3}$ is a (multiplicative) set of generators of $G_{\boldsymbol{e}}=$ $T_{e}=T_{c}=G_{c}$. 
(ii) Next suppose that $G_{\boldsymbol{c}}=G_{\boldsymbol{e}}$ and $k_{c}=k_{e}$ is odd. Then the conditions of the previous proposition hold and $T_{\boldsymbol{c}}=T_{\boldsymbol{e}}$. We have that

$$
G_{\boldsymbol{c}}=T_{\boldsymbol{c}} \cup \boldsymbol{c}_{1} T_{\boldsymbol{c}} \cup \boldsymbol{c}_{2} T_{\boldsymbol{c}} \cup \boldsymbol{c}_{1} \boldsymbol{c}_{2} T_{\boldsymbol{c}},
$$

from Cermelli and Parry [5]. Let the four cosets in $G_{\boldsymbol{e}} / T_{\boldsymbol{e}}$ be $T_{\boldsymbol{e}}, \boldsymbol{\alpha} T_{\boldsymbol{e}}, \boldsymbol{\beta} T_{\boldsymbol{e}}, \boldsymbol{\gamma} T_{\boldsymbol{e}}$ for some $\boldsymbol{\alpha}, \boldsymbol{\beta}, \boldsymbol{\gamma} \in G_{\boldsymbol{e}}$. It is necessary, then, that $T_{\boldsymbol{c}}=T_{\boldsymbol{e}}$ and that $\boldsymbol{c}_{1} T_{\boldsymbol{c}}, \boldsymbol{c}_{2} T_{\boldsymbol{c}}$ and $\boldsymbol{c}_{1} \boldsymbol{c}_{2} T_{\boldsymbol{c}}$ are the same as $\boldsymbol{\alpha} T_{\boldsymbol{e}}, \boldsymbol{\beta} T_{\boldsymbol{e}}, \boldsymbol{\gamma} T_{\boldsymbol{e}}$, in some order. Note that if $\boldsymbol{c}_{1} T_{\boldsymbol{c}}, \boldsymbol{c}_{2} T_{\boldsymbol{c}}$ are some two of $\boldsymbol{\alpha} T_{\boldsymbol{e}}, \boldsymbol{\beta} T_{\boldsymbol{e}}, \boldsymbol{\gamma} T_{\boldsymbol{e}}$, then $\boldsymbol{c}_{1} \boldsymbol{c}_{2} T_{\boldsymbol{c}}$ is the one remaining coset of those three. Notice that $T_{\boldsymbol{c}}=T_{\boldsymbol{e}}$ if and only if

$$
\left\langle 2 c_{1}, 2 c_{2}, c_{3}\right\rangle=\left\langle 2 e_{1}, 2 e_{2}, 2 e_{3}, \frac{2 \nu_{i} e_{i}}{\Gamma}\right\rangle .
$$

Consider first the constraints on representatives $\mathbf{0}, \boldsymbol{\alpha}, \boldsymbol{\beta}, \boldsymbol{\gamma}$ of the four distinct cosets in $G_{\boldsymbol{e}} / T_{\boldsymbol{e}}$. According to Cermelli and Parry [5] and Parry [14], all elements $\boldsymbol{\alpha} \in$ $G_{\boldsymbol{e}}$ have the form $\boldsymbol{\alpha}=\boldsymbol{e}_{1}^{l_{1}} \boldsymbol{e}_{2}^{l_{2}} \boldsymbol{e}_{3}^{l_{3}} \boldsymbol{c}_{3}^{l_{0}}$, for some integers $l_{0}, l_{1}, l_{2}, l_{3}$ (writing $\boldsymbol{c}_{3}$ for a generator of $\left.\mathbb{Z}\left(G_{\boldsymbol{e}}\right)=\mathbb{Z}\left(G_{\boldsymbol{c}}\right)\right)$. If $\boldsymbol{\alpha}$ has this form, and correspondingly $\boldsymbol{\beta}=$ $\boldsymbol{e}_{1}^{m_{1}} \boldsymbol{e}_{2}^{m_{2}} \boldsymbol{e}_{3}^{m_{3}} \boldsymbol{c}_{3}^{m_{0}}$, for integers $m_{0}, m_{1}, m_{2}, m_{3}$, then from [5], $\boldsymbol{\alpha}$ and $\boldsymbol{\beta}$ are in the same coset if and only if

$$
l_{i}-m_{i}=\tau \nu_{i}, \quad \bmod 2, \quad i=1,2,3,
$$

for some integer $\tau$. Let $\bar{l}=0$ if $l$ is even, $\bar{l}=1$ if $l$ is odd. Then (55) is equivalent to

$$
\bar{l}_{i}-\bar{m}_{i}=\overline{\tau \nu}_{i}=\bar{\tau} \bar{\nu}_{i}=0 \quad \text { or } \quad \bar{\nu}_{i}, \quad \bmod 2 .
$$

Note that (56) is also equivalent to the condition that

$$
r l_{i}+s m_{i}=\tau \nu_{i}, \quad \bmod 2,
$$

for some integers $r, s, \tau$, as this also reduces to the requirement that either two of $\bar{l}_{i}, \bar{m}_{i}, \bar{\nu}_{i}$ are the same (for all $i=1,2,3$ ), or that any one of $\bar{l}_{i}, \bar{m}_{i}, \bar{\nu}_{i}$ is the sum (or difference) of the other two, for all $i=1,2,3$. Bearing in mind that $\boldsymbol{e}_{1}^{\nu_{1}} \boldsymbol{e}_{2}^{\nu_{2}} \boldsymbol{e}_{3}^{\nu_{3}} \boldsymbol{c}_{3}^{\nu_{0}} \in$ $\mathbb{Z}\left(G_{\boldsymbol{e}}\right) \subset T_{\boldsymbol{e}}$, it follows that $T_{\boldsymbol{e}}, \boldsymbol{\alpha} T_{\boldsymbol{e}}, \boldsymbol{\beta} T_{\boldsymbol{e}}$ are different cosets, in $G_{\boldsymbol{e}} / T_{\boldsymbol{e}}$, if and only if the rows, $\boldsymbol{l}=\left(l_{1}, l_{2}, l_{3}\right), \boldsymbol{m}=\left(m_{1}, m_{2}, m_{3}\right), \boldsymbol{\nu}=\left(\nu_{1}, \nu_{2}, \nu_{3}\right)$, of the matrix $A$ are linearly independent, mod 2 .

It follows that, if $G_{\boldsymbol{c}}=G_{\boldsymbol{e}}$, then $\boldsymbol{c}_{1} \in \boldsymbol{\alpha} T_{\boldsymbol{e}}, \boldsymbol{c}_{2} \in \boldsymbol{\beta} T_{\boldsymbol{e}}$ for $\boldsymbol{\alpha}, \boldsymbol{\beta} \in G_{\boldsymbol{e}}$ corresponding to triples $\boldsymbol{l}, \boldsymbol{m}$ such that $A$ is an integer matrix whose rows are linearly independent $\bmod 2$.

Since

$$
\begin{aligned}
\boldsymbol{\alpha} T_{\boldsymbol{e}} & =\boldsymbol{\alpha}+T_{\boldsymbol{e}}=\boldsymbol{e}_{1}^{l_{1}} \boldsymbol{e}_{2}^{l_{2}} \boldsymbol{e}_{3}^{l_{3}} \boldsymbol{c}_{3}^{l_{0}}+T_{\boldsymbol{e}} \\
& =\bar{l}_{1} \boldsymbol{e}_{1}+\bar{l}_{2} \boldsymbol{e}_{2}+\bar{l}_{3} \boldsymbol{e}_{3}+\frac{1}{2} \theta(\overline{\boldsymbol{l}}) \boldsymbol{c}_{3}+T_{\boldsymbol{e}}
\end{aligned}
$$

where the definition of $\theta(\bar{\ell})$ is given in the statement of the theorem, it follows that there are integers $L_{1}, L_{2}, L_{3}$ with the same parity as $\ell_{1}, \ell_{2}, \ell_{3}$, respectively, such that

$$
\boldsymbol{c}_{1}=L_{1} \boldsymbol{e}_{1}+L_{2} \boldsymbol{e}_{2}+L_{3} \boldsymbol{e}_{3}+\frac{1}{2} \theta(\overline{\boldsymbol{L}}) \boldsymbol{c}_{3}+l_{0} \boldsymbol{c}_{3} .
$$

Without loss of generality, we write $l_{1}, l_{2}, l_{3}$ for $L_{1}, L_{2}, L_{3}$, etc., and have:

$$
\begin{aligned}
\boldsymbol{c}_{1} & =l_{1} \boldsymbol{e}_{1}+l_{2} \boldsymbol{e}_{2}+l_{3} \boldsymbol{e}_{3}+\frac{1}{2} \theta(\overline{\boldsymbol{l}}) \boldsymbol{c}_{3}+l_{0} \boldsymbol{c}_{3}, \\
\boldsymbol{c}_{2} & =m_{1} \boldsymbol{e}_{1}+m_{2} \boldsymbol{e}_{2}+m_{3} \boldsymbol{e}_{3}+\frac{1}{2} \theta(\overline{\boldsymbol{m}}) \boldsymbol{c}_{3}+m_{0} \boldsymbol{c}_{3}, \\
\pm \frac{\Gamma}{2} \boldsymbol{c}_{3} & =\nu_{1} \boldsymbol{e}_{1}+\nu_{2} \boldsymbol{e}_{2}+\nu_{3} \boldsymbol{e}_{3} .
\end{aligned}
$$


This gives equation (48) in the statement of the theorem, except that we have no information regarding $\operatorname{det} A$ yet. From (60), comparing with $2 \boldsymbol{e}_{i}=\gamma_{i j} \boldsymbol{c}_{j}^{\prime}$, we get

$$
\gamma=A^{-1}\left(\begin{array}{ccc}
1 & 0 & -\left(2 l_{0}+\theta(\boldsymbol{l})\right) \\
0 & 1 & -\left(2 m_{0}+\theta(\boldsymbol{m})\right) \\
0 & 0 & \pm \Gamma
\end{array}\right)
$$

and it follows that $\operatorname{det} \gamma=\operatorname{det} A^{-1}( \pm \Gamma)=\Gamma$, so $\operatorname{det} A= \pm 1$ and $A \in G L_{3}(\mathbb{Z})$. This condition is itself sufficient that the rows of $A$ as linearly independent, mod 2 (for if one adds two to a single element of $A$, then $\operatorname{det} A$ is changed by an even number). So the necessity of (48) is proven.

Conversely, if (48) holds, then the group elements corresponding to the rows $\boldsymbol{l}, \boldsymbol{m}, \boldsymbol{\nu}$ of $A$ are in different cosets, in $G_{\boldsymbol{e}} / T_{\boldsymbol{e}}$. Multiplying the first two equations of (60), or (48), by two, one sees that (48) implies that $T_{\boldsymbol{c}}=T_{\boldsymbol{e}}$ (via (54)). The first equation of (60) gives $\boldsymbol{c}_{1} \in \boldsymbol{\alpha} T_{\boldsymbol{e}}$, so $\boldsymbol{c}_{1}$ is a product of $\boldsymbol{\alpha} \in G_{\boldsymbol{e}}$ with an integer linear combination of $2 \boldsymbol{e}_{1}, 2 \boldsymbol{e}_{1}, 2 \boldsymbol{e}_{3}, 2 \nu_{i} \boldsymbol{e}_{i} / \Gamma$. But $2 \nu_{i} \boldsymbol{e}_{i} / \Gamma$ generates $\mathbb{Z}\left(G_{\boldsymbol{e}}\right)$ (by the conditions which appear in proposition 6 , for they guarantee that $\mathbb{Z}\left(G_{\boldsymbol{e}}\right)=\mathbb{Z}\left(G_{\boldsymbol{c}}\right)$, and $\mathbb{Z}\left(G_{\boldsymbol{c}}\right)$ is generated by $\left.\boldsymbol{c}_{3}=2 \nu_{i} \boldsymbol{e}_{i} / \Gamma\right)$, and so is a product of $\boldsymbol{e}_{1}, \boldsymbol{e}_{2}, \boldsymbol{e}_{3}$ and their inverses. Hence $\boldsymbol{c}_{1}$ is expressible as a product of $\boldsymbol{e}_{1}, \boldsymbol{e}_{2}, \boldsymbol{e}_{3}$ and their inverses. Likewise for $\boldsymbol{c}_{2}$. This proves that $\boldsymbol{e}_{1}, \boldsymbol{e}_{2}, \boldsymbol{e}_{3}$ is a (multiplicative) set of generators for $G_{c}$.

\section{Generalization of Ericksen-Pitteri neighbourhoods}

Let $\boldsymbol{c}_{1}, \boldsymbol{c}_{2}, \boldsymbol{c}_{3}$ be a canonical basis of a discrete subgroup $G_{\boldsymbol{c}} \subset J$ such that (30) holds for some $k \in \mathbb{Z}$. With respect to this basis the Lie bracket associated with this subgroup is given by (33) and hence the corresponding structure constants are $C_{i j k}=\varepsilon_{3 j k} k \delta_{i 3}$ and by (9) the components of the dislocation density tensor, $S$, with respect to this basis are $S_{p r}=k \delta_{p 3} \delta_{r 3}$.

Let $\boldsymbol{e}_{1}, \boldsymbol{e}_{2}, \boldsymbol{e}_{3}$ be a basis of $\mathbb{R}^{3}$ which satisfies the conditions of Theorem 7 so that $G_{\boldsymbol{e}}=G_{\boldsymbol{c}}$ where $G_{\boldsymbol{e}}$ is the group generated by $\boldsymbol{e}_{1}, \boldsymbol{e}_{2}, \boldsymbol{e}_{3}$. By Proposition 4 , with respect to the basis $\boldsymbol{e}_{1}, \boldsymbol{e}_{2}, \boldsymbol{e}_{3}$, the Lie bracket has the form (35) and hence the components of the dislocation density tensor with respect to this basis are $S_{p r}=\frac{\theta k}{T} \nu_{p} \nu_{r}$ where the integers $k, \Gamma, \nu_{p}, p=1,2,3$ satisfy the conditions of Proposition 6 .

Since $G_{\boldsymbol{c}}$ and $G_{\boldsymbol{e}}$ are the same group they correspond to the same set of points in $\mathbb{R}^{3}$ and hence if $w$ is a strain energy function then that depends only on the positions of these points, then

$$
w\left(\left\{\boldsymbol{e}_{i}\right\}, S_{p r}=\frac{\theta k}{T} \nu_{p} \nu_{r}\right)=w\left(\left\{\boldsymbol{c}_{i}\right\}, S_{p r}=k \delta_{p 3} \delta_{r 3}\right)
$$

where the $\boldsymbol{e}_{i}, i=1,2,3$ can be expressed in terms of the $\boldsymbol{c}_{i}, i=1,2,3$ and vice versa via (47) or (48) depending on the parity of $k$. 
Suppose now that $\boldsymbol{c}_{1}^{*}, \boldsymbol{c}_{2}^{*}, \boldsymbol{c}_{3}^{*} \in \mathbb{R}^{3}$ is a canonical basis of another discrete subgroup $G_{\boldsymbol{c}^{*}} \subset J$ and that $G_{\boldsymbol{c}^{*}}=G_{\boldsymbol{e}^{*}}$ where $G_{\boldsymbol{e}^{*}}$ is generated by $\boldsymbol{e}_{1}^{*}, \boldsymbol{e}_{2}^{*}, \boldsymbol{e}_{3}^{*} \in G_{\boldsymbol{c}^{*}}$. Then we also have

$$
w\left(\left\{\boldsymbol{e}_{i}^{*}\right\}, S_{p r}^{*}=\frac{\theta^{*} k^{*}}{\Gamma^{*}} \nu_{p}^{*} \nu_{r}^{*}\right)=w\left(\left\{\boldsymbol{c}_{i}^{*}\right\}, S_{p r}^{*}=k^{*} \delta_{p 3} \delta_{r 3}\right),
$$

where the integers $k^{*}, \Gamma^{*}, \nu_{p}^{*}, p=1,2,3$ also satisfy the conditions of Proposition 6 and the $\boldsymbol{e}_{i}^{*}, i=1,2,3$ can be expressed in terms of the $\boldsymbol{c}_{j}^{*}, j=1,2,3$ and vice versa via (47) or (48) depending on the parity of $k^{*}$.

Equations (62), (63) express some global symmetries of a strain energy function that depends only on the positions of points that correspond to the elements of a given discrete subgroup. In what follows we shall see that a given set of points may correspond to different subgroups $G_{\boldsymbol{c}}, G_{\boldsymbol{c}^{*}}$, with $k \neq k^{*}$, so that (62) and (63) must be supplemented by further conditions so as to reflect the fact that the energy density is to be independent of the description of the given set of points in terms of any particular choice of group, and any particular choice of generators within a group. However to begin with we deal with the simplest case where $G_{\boldsymbol{c}}=G_{\boldsymbol{c}^{*}}, k=k^{*}$ and the relevant symmetries derive just from (62) and (63), and prove an analogue of the EricksenPitteri result (cf. (i) and (ii) in the Introduction). The remainder of the section is devoted to the case where $k \neq k^{*}$.

$4.1 k=k^{*}, G_{\boldsymbol{c}}=G_{c^{*}}$

Suppose that $k=k^{*}$ with $\left\{\boldsymbol{c}_{i}\right\}=\left\{\boldsymbol{c}_{i}^{*}\right\}$ a canonical basis of $J$ such that $\left(\boldsymbol{c}_{1}, \boldsymbol{c}_{2}\right)=$ $\boldsymbol{c}_{3}^{k},\left(\boldsymbol{c}_{2}, \boldsymbol{c}_{3}\right)=\left(\boldsymbol{c}_{1}, \boldsymbol{c}_{3}\right)=\mathbf{0}$. We then have from (62) and (63), since $k=k^{*}$ and since $\theta$ depends only on the parity of $k, \theta=\theta^{*}$,

$$
w\left(\left\{\boldsymbol{e}_{i}\right\}, S_{p r}=\frac{\theta k}{\Gamma} \nu_{p} \nu_{r}\right)=w\left(\left\{\boldsymbol{e}_{i}^{*}\right\}, S_{p r}^{*}=\frac{\theta k}{\Gamma^{*}} \nu_{p}^{*} \nu_{r}^{*}\right) .
$$

Then from (47) and (48), since $\boldsymbol{e}_{1}, \boldsymbol{e}_{2}, \boldsymbol{e}_{3}$ and $\boldsymbol{e}_{1}^{*}, \boldsymbol{e}_{2}^{*}, \boldsymbol{e}_{3}^{*}$ are two different sets of generators of the group $G_{\boldsymbol{e}}=G_{\boldsymbol{c}}=G_{c^{*}}=G_{e^{*}}$, we have for $k$ even

$$
\begin{aligned}
& \left(\begin{array}{c}
\boldsymbol{e}_{1}^{*} \\
\boldsymbol{e}_{2}^{*} \\
\boldsymbol{e}_{3}^{*}
\end{array}\right)=\left(A^{*}\right)^{-1}\left(\begin{array}{ccc}
1 & 0 & -l_{0}^{*} \\
0 & 1 & -m_{0}^{*} \\
0 & 0 & \pm \Gamma^{*}
\end{array}\right)\left(\begin{array}{l}
c_{1}^{*} \\
\boldsymbol{c}_{2}^{*} \\
\boldsymbol{c}_{3}^{*}
\end{array}\right)=\left(A^{*}\right)^{-1}\left(\begin{array}{ccc}
1 & 0 & -l_{0}^{*} \\
0 & 1 & -m_{0}^{*} \\
0 & 0 & \pm \Gamma^{*}
\end{array}\right)\left(\begin{array}{l}
\boldsymbol{c}_{1} \\
\boldsymbol{c}_{2} \\
\boldsymbol{c}_{3}
\end{array}\right)
\end{aligned}
$$

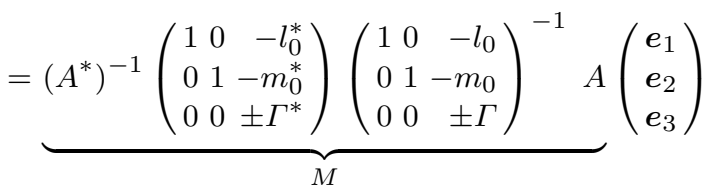

and for $k$ odd,

$$
\begin{aligned}
\left(\begin{array}{c}
\boldsymbol{e}_{1}^{*} \\
\boldsymbol{e}_{2}^{*} \\
\boldsymbol{e}_{3}^{*}
\end{array}\right) & =\left(A^{*}\right)^{-1}\left(\begin{array}{ccc}
1 & 0 & -\left(2 l_{0}^{*}+\theta\left(\boldsymbol{l}^{*}\right)\right) \\
0 & 1 & -\left(2 m_{0}^{*}+\theta\left(\boldsymbol{m}^{*}\right)\right) \\
0 & 0 & \pm \Gamma^{*}
\end{array}\right)\left(\begin{array}{c}
\boldsymbol{c}_{1}^{*} \\
\boldsymbol{c}_{2}^{*} \\
\frac{1}{2} \boldsymbol{c}_{3}^{*}
\end{array}\right) \\
& =\underbrace{\left(A^{*}\right)^{-1}\left(\begin{array}{ccc}
1 & 0 & -\left(2 l_{0}^{*}+\theta\left(\boldsymbol{l}^{*}\right)\right) \\
0 & 1 & -\left(2 m_{0}^{*}+\theta\left(\boldsymbol{m}^{*}\right)\right) \\
0 & 0 & \pm \Gamma^{*}
\end{array}\right)\left(\begin{array}{ccc}
1 & 0 & -\left(2 l_{0}+\theta(\boldsymbol{l})\right) \\
0 & 1 & -\left(2 m_{0}+\theta(\boldsymbol{m})\right) \\
0 & 0 & \pm \Gamma
\end{array}\right)^{-1} A}_{M}\left(\begin{array}{l}
\boldsymbol{e}_{1} \\
\boldsymbol{e}_{2} \\
\boldsymbol{e}_{3}
\end{array}\right),
\end{aligned}
$$


where $A, A^{*} \in G L_{3}(\mathbb{Z})$ and

$$
\operatorname{det}\left\{\boldsymbol{e}_{i}^{*}\right\}=\Gamma^{*} \operatorname{det}\left\{\boldsymbol{c}_{i}^{*}\right\}=\frac{\Gamma^{*}}{\Gamma} \Gamma \operatorname{det}\left\{\boldsymbol{c}_{i}\right\}=\frac{\Gamma^{*}}{\Gamma} \operatorname{det}\left\{\boldsymbol{e}_{i}\right\}
$$

In (65) and (66), let

$$
\boldsymbol{e}_{i}^{*} \equiv M_{i j} \boldsymbol{e}_{j}, \quad i, j=1,2,3 .
$$

Let the third row of $A$ be $\left(\nu_{1}, \nu_{2}, \nu_{3}\right)$, and let the third row of $A^{*}$ be $\left(\nu_{1}^{*}, \nu_{2}^{*}, \nu_{3}^{*}\right)$, with the elements of each row consisting of relatively prime integers (as $A^{*}, A \in G L_{3}(\mathbb{Z})$ ). We have from (65)-(68),

$$
\operatorname{det} M=\frac{\Gamma^{*}}{\Gamma}, \quad M_{i j}=\frac{m_{i j}}{\Gamma} \text { for some } m_{i j} \in \mathbb{Z}, \quad i, j=1,2,3 .
$$

From Theorem 7, (65)-(68), we get

$$
M_{i j} \nu_{i}^{*}=\tau \frac{\Gamma^{*}}{\Gamma} \nu_{j} \text {, where } \tau \equiv \operatorname{sign}\left(\operatorname{det} A^{*}\right) \operatorname{sign}(\operatorname{det} A) .
$$

Now define

$$
\begin{aligned}
\mathcal{H}\left(k, \Gamma,\left\{\nu_{i}\right\}\right) & \equiv\left\{M \in G L_{3}(\mathbb{Q}):\left\{\boldsymbol{e}_{i}^{*}=M_{i j} \boldsymbol{e}_{j}\right\} \text { generates } G_{\boldsymbol{e}}\right\} \\
& =\left\{M \in G L_{3}(\mathbb{Q}): M \text { is as in }(65),(66)\right\}
\end{aligned}
$$

Notice that if $M, M^{\prime} \in \mathcal{H}\left(k, \Gamma,\left\{\nu_{i}\right\}\right)$, and $M \neq M^{\prime}$, then there exist indices $i, j \in$ $\{1,2,3\}$ such that (with $M^{\prime}=\left(M_{i j}^{\prime}\right) \equiv\left(m_{i j}^{\prime} / \Gamma\right)$ )

$$
\left|M_{i j}^{\prime}-M_{i j}\right| \geq 1 /|\Gamma| \text {. }
$$

Now any frame indifferent strain energy function $w$ must satisfy $w\left(\left\{\boldsymbol{e}_{i}\right\}, S\right)=$ $w\left(\left\{\mathcal{R}_{i}\right\}, S\right)$ for any rotation $\mathcal{R}$, so that $w\left(\left\{\boldsymbol{e}_{i}\right\}, S\right)=\tilde{w}(C, S)$ where $C$ is the positive definite symmetric matrix with entries $C_{i j}=\boldsymbol{e}_{i} \cdot \boldsymbol{e}_{j} . C$ is the metric of the basis $\boldsymbol{e}_{1}, \boldsymbol{e}_{2}, \boldsymbol{e}_{3}$ and $C=E^{T} E$ where $E$ is the matrix with columns $\boldsymbol{e}_{1}, \boldsymbol{e}_{2}, \boldsymbol{e}_{3}$. Defining the metric $C^{*}$ similarly, from (64) we have $\tilde{w}(C, S)=\tilde{w}\left(C^{*}, S^{*}\right)$. Note that since $\boldsymbol{e}_{i}^{*}=M_{i j} \boldsymbol{e}_{j}$, if $E^{*}$ is the matrix with columns $\boldsymbol{e}_{1}^{*}, \boldsymbol{e}_{2}^{*}, \boldsymbol{e}_{3}^{*},\left(E^{*}\right)^{T}=M E^{T}$ and thus

$$
C^{*}=\left(E^{*}\right)^{T} E^{*}=M E^{T}\left(M E^{T}\right)^{T}=M E^{T} E M^{T}=M C M^{T} .
$$

We want to determine, in part, whether or not if $(C, S)$ is given, the quantities $\left(C^{*}, S^{*}\right)$ may accumulate at $(C, S)$. To proceed, we adapt Ball and James [2] a little and introduce a neighbourhood $N_{\varepsilon}, \varepsilon>0$, of the point $\left(\left\{\boldsymbol{e}_{i}\right\}, S\right)$ via the following definitions:

$$
\begin{aligned}
& N_{\varepsilon}\left(\left\{\boldsymbol{e}_{i}\right\}, k, \Gamma,\left\{\nu_{i}\right\}\right) \equiv \\
& \left\{\left(\left\{\boldsymbol{e}_{i}^{*}\right\},\left\{\frac{k^{*}}{\Gamma^{*}} \nu_{i}^{*} \nu_{j}^{*}\right\}\right):\left\|C^{*}-C\right\|_{\left\{\boldsymbol{e}_{i}\right\}}<\varepsilon,\left\|\frac{k^{*}}{\Gamma^{*}} \nu_{i}^{*} \nu_{j}^{*}-\frac{k}{\Gamma} \nu_{i} \nu_{j}\right\|<\varepsilon\right\},
\end{aligned}
$$

where $\|A\| \geq 0$ is defined by

$$
\|A\|^{2} \equiv \operatorname{tr}\left(A^{T} A\right)
$$

and

$$
\|A\|_{\left\{\boldsymbol{e}_{i}\right\}}=\left\|D A D^{T}\right\|,
$$

where $E$ is the matrix with columns $\boldsymbol{e}_{1}, \boldsymbol{e}_{2}, \boldsymbol{e}_{3}$ and $D \equiv E^{-T}$. 
Proposition 8 If $\varepsilon$ is small enough, $k \neq 0$ and some $\nu_{i} \neq 0$, then $\left(\left\{\boldsymbol{e}_{i}^{*}\right\},\left\{\frac{k^{*}}{\Gamma^{*}} \nu_{i}^{*} \nu_{j}^{*}\right\}\right)$ $\in N_{\varepsilon}\left(\left\{\boldsymbol{e}_{i}\right\}, k, \Gamma,\left\{\nu_{i}\right\}\right)$ only if $\Gamma=\Gamma^{*}, \nu_{i}^{*}= \pm \nu_{i}$ for $i=1,2,3$ (with the choice of sign independent of the index $i)$.

\section{Proof}

First, if $\left\|C^{*}-C\right\|_{\left\{\boldsymbol{e}_{i}\right\}}=\left\|D C^{*} D^{T}-D C D^{T}\right\|$ is sufficiently small, then $\operatorname{det} D C^{*} D^{T}$ can be made arbitrarily close to $\operatorname{det} D C D^{T}$. Hence, as $\operatorname{det} D \neq 0$ is fixed, $\operatorname{det} C^{*}=$ $\operatorname{det} C(\operatorname{det} M)^{2}$ is arbitrarily close to $\operatorname{det} C$, and so from $(69),\left(\Gamma^{*}\right)^{2}=\Gamma^{2}$, since both $\Gamma^{*}, \Gamma$ are integers.

Next, if $\left\|\frac{k}{\Gamma^{*}} \nu_{i}^{*} \nu_{j}^{*}-\frac{k}{\Gamma} \nu_{i} \nu_{j}\right\|=\left|\frac{k}{\Gamma}\right|\left\|\nu_{i}^{*} \nu_{j}^{*} \pm \nu_{i} \nu_{j}\right\|$ is sufficiently small (recall $k=k^{*}$ in this section), then $\nu_{i}^{*}= \pm \nu_{i}, i=1,2,3$, with the choice of sign possibly dependent on the index $i$. (For if this were not so, $\nu_{i}^{* 2} \neq \nu_{i}^{2}$ for some index $i$ and this would contradict the fact that $\left|\frac{k}{\Gamma}\right|\left|\nu_{i}^{* 2} \pm \nu_{i}^{2}\right| \geq\left|\frac{k}{\Gamma}\right|$ is sufficiently small).

Then, if for example $\nu_{1}^{*}=\nu_{1}, \nu_{2}^{*}=-\nu_{2}$ with $\nu_{1} \nu_{2} \neq 0$, in the case $\Gamma=\Gamma^{*}$ we get $\left|\frac{k}{\Gamma}\right||| \nu_{i}^{*} \nu_{j}^{*}-\nu_{i} \nu_{j} \| \geq\left|\frac{k}{\Gamma}\right|\left|2 \nu_{1} \nu_{2}\right| \geq\left|\frac{k}{\Gamma}\right|$, whereas in the case $\Gamma=-\Gamma^{*}$ one has $\left|\frac{k}{\Gamma}\right||| \nu_{i}^{*} \nu_{j}^{*}+\nu_{i} \nu_{j} \| \geq\left|\frac{k}{\Gamma}\right|\left|2 \nu_{1}^{2}\right| \geq\left|\frac{k}{\Gamma}\right|$. In either case we get a contradiction, so that $\nu_{i}^{*}= \pm \nu_{i}$, with sign independent of the index. Finally, with this information, $\left\|\frac{k}{\Gamma^{*}} \nu_{i}^{*} \nu_{j}^{*}-\frac{k}{\Gamma} \nu_{i} \nu_{j}\right\|=\left|\frac{k}{\Gamma}\right|\left|\left(\operatorname{sign} \frac{\Gamma}{\Gamma^{*}}-1\right)\right|\left\|\nu_{i} \nu_{j}\right\|$ leads to a contradiction unless $\Gamma=$ $\Gamma^{*}$. This proves the proposition.

\section{Definition}

$$
\mathcal{L}\left(k, \Gamma,\left\{\nu_{i}\right\}\right) \equiv\left\{M \in \mathcal{H}\left(k, \Gamma,\left\{\nu_{i}\right\}\right): \Gamma^{*}=\Gamma \text { and } \nu_{i}^{*}= \pm \nu_{i}, \quad i=1,2,3\right\},
$$

when the sign in the expression $\pm \nu_{i}$ is independent of the index $i$. We remark that $\mathcal{L}\left(k, \Gamma,\left\{\nu_{i}\right\}\right)$ is a subgroup of $G L_{3}(\mathbb{Q})$.

Proposition 9 The free substitution of $G_{\boldsymbol{e}}$ which takes generators $\left\{\boldsymbol{e}_{i}\right\}$ to generators $\left\{\boldsymbol{e}_{i}^{*}\right\}$ (corresponding to $\boldsymbol{e}_{i}^{*}=M_{i j} \boldsymbol{e}_{j}$, with $\left(M_{i j}\right)$ the matrices that appear in (65) and (66)) extends to an elastic deformation of $J$ if and only if $\frac{k}{\Gamma^{*}} \nu_{i}^{*} \nu_{j}^{*}=\frac{k}{\Gamma} \nu_{i} \nu_{j}$.

\section{Proof}

Let $\boldsymbol{\psi}: G_{\boldsymbol{e}} \rightarrow G_{\boldsymbol{e}}$ be the free substitution defined by $\boldsymbol{\psi}\left(\boldsymbol{e}_{i}\right)=\boldsymbol{e}_{i}^{*}, i=1,2,3$, where $\boldsymbol{e}_{i}^{*}=M_{i j} \boldsymbol{e}_{j}$ in line with (65), (66). Then according to Auslander [1], Parry [12], $\boldsymbol{\psi}$ extends to an elastic deformation (automorphism) of $J$ if and only if the defining relations of $G_{\boldsymbol{e}}$ are invariant under that substitution and its inverse, i.e., if and only if

$$
\left(\boldsymbol{\psi}\left(\boldsymbol{e}_{i}\right), \boldsymbol{\psi}\left(\boldsymbol{e}_{j}\right)\right)=\boldsymbol{\psi}\left(\varepsilon_{i j p} k \nu_{p} \boldsymbol{c}_{3}\right), \quad\left(\boldsymbol{\psi}^{-1}\left(\boldsymbol{e}_{i}\right), \boldsymbol{\psi}^{-1}\left(\boldsymbol{e}_{j}\right)\right)=\boldsymbol{\psi}^{-1}\left(\varepsilon_{i j p} k \nu_{p} \boldsymbol{c}_{3}\right),
$$

where, in (78), $\boldsymbol{c}_{3}$ is a product of the elements $\boldsymbol{e}_{1}, \boldsymbol{e}_{2}, \boldsymbol{e}_{3}$ that generates $\mathbb{Z}\left(G_{\boldsymbol{e}}\right)$. Note that $\boldsymbol{\psi}^{-1}$ is the substitution that maps $\boldsymbol{\psi}\left(\boldsymbol{e}_{i}\right)$ to $\boldsymbol{e}_{i}, i=1,2,3$. Thus we have to show just that (78) is equivalent to $\Gamma=\Gamma^{*}, \nu_{i}^{*}= \pm \nu_{i}$ (bearing in mind that $\frac{1}{\Gamma^{*}} \nu_{i}^{*} \nu_{j}^{*}=\frac{1}{\Gamma} \nu_{i} \nu_{j}$ is equivalent to $\Gamma=\Gamma^{*}, \nu_{i}^{*}= \pm \nu_{i}$, when $\left\{\nu_{i}^{*}\right\},\left\{\nu_{i}\right\}$ are triples of relatively prime integers). 
First suppose that $(78)_{1}$ holds. Then

$$
\begin{aligned}
\left(\boldsymbol{\psi}\left(\boldsymbol{e}_{i}\right), \boldsymbol{\psi}\left(\boldsymbol{e}_{j}\right)\right)=\left[M_{i p} \boldsymbol{e}_{p}, M_{j q} \boldsymbol{e}_{q}\right] & =M_{i p} M_{j q} \varepsilon_{p q r} k \nu_{r} \boldsymbol{c}_{3} \\
& =\varepsilon_{i j \ell} M_{r \ell}^{-1} \operatorname{det}(M) k \nu_{r} \boldsymbol{c}_{3}=\varepsilon_{i j \ell} \tau \nu_{\ell}^{*} k \boldsymbol{c}_{3},
\end{aligned}
$$

from (70) and (71). Since $\varepsilon_{i j p} k \nu_{p}$ is an integer,

$$
\boldsymbol{\psi}\left(\varepsilon_{i j p} k \nu_{p} \boldsymbol{c}_{3}\right)=\varepsilon_{i j p} k \nu_{p} \boldsymbol{\psi}\left(\boldsymbol{c}_{3}\right)
$$

Since $\boldsymbol{\psi}$ is an automorphism, $\boldsymbol{\psi}\left(\boldsymbol{c}_{3}\right)$ generates $\mathbb{Z}\left(G_{\boldsymbol{e}}\right)$, as does $\boldsymbol{c}_{3}$, so

$$
\boldsymbol{\psi}\left(\boldsymbol{c}_{3}\right)=\boldsymbol{c}_{3}^{ \pm 1}=\varepsilon \boldsymbol{c}_{3}, \text { say, where } \varepsilon= \pm 1
$$

So from (79), (80), (81)

$$
\tau \nu_{\ell}^{*}=\varepsilon \nu_{\ell}
$$

From Parry [12], following Mal'cev [10], as $\boldsymbol{\psi}: G_{\boldsymbol{e}} \rightarrow G_{\boldsymbol{e}}$ is an automorphism, it extends uniquely to a linear automorphism $\phi: J \rightarrow J$. In the case $k$ even, from (39), $c_{3}=\frac{\pi}{T} \nu_{k} e_{k}$, where $\pi= \pm 1$, so

$$
\begin{aligned}
\varepsilon \boldsymbol{c}_{3} & =\boldsymbol{\psi}\left(\boldsymbol{c}_{3}\right)=\boldsymbol{\psi}\left(\frac{\pi}{\Gamma} \nu_{k} \boldsymbol{e}_{k}\right)=\boldsymbol{\phi}\left(\frac{\pi}{\Gamma} \nu_{k} \boldsymbol{e}_{k}\right)=\frac{\pi}{\Gamma} \nu_{k} \phi\left(\boldsymbol{e}_{k}\right)=\frac{\pi}{\Gamma} \nu_{k} \boldsymbol{e}_{k}^{*} \\
& =\frac{\pi}{\Gamma} \nu_{k} M_{k j} \boldsymbol{e}_{j}=\frac{\pi \varepsilon \tau}{\Gamma} \nu_{k}^{*} M_{k j} \boldsymbol{e}_{j}=\varepsilon \pi \frac{\Gamma^{*}}{\Gamma^{2}} \nu_{j} \boldsymbol{e}_{j}
\end{aligned}
$$

via (70). Hence

$$
\varepsilon \boldsymbol{c}_{3} \equiv \varepsilon\left(\frac{\pi}{\Gamma} \nu_{k} \boldsymbol{e}_{k}\right)=\varepsilon \pi \frac{\Gamma^{*}}{\Gamma^{2}} \nu_{j} \boldsymbol{e}_{j}, \text { so } \Gamma=\Gamma^{*} .
$$

Equations (82) and (84) show that $\nu_{\ell}^{*}= \pm \nu_{\ell}, \Gamma^{*}=\Gamma$.

Since $\boldsymbol{\psi}^{-1}$ is also an automorphism, it also extends to a (linear) automorphism of $J$ and so corresponds to the linear transformation $M^{-1}$. One checks that $(78)_{2}$ provides no further information, in this case. The case where $k$ is odd is similar, and we leave it to the reader to check that if $\nu_{\ell}^{*}= \pm \nu_{\ell}, \Gamma^{*}=\Gamma$, then (78) holds.

Propositions 8 and 9, taken together, imply that the symmetries of this class of defective crystals which are sufficiently small (in the sense of (74)) extend to elastic deformations. In particular, if $\varepsilon$ is sufficiently small then (cf. (74))

$$
N_{\varepsilon}\left(\left\{\boldsymbol{e}_{i}\right\}, k, \Gamma,\left\{\nu_{i}\right\}\right)=\left\{\left(\left\{\boldsymbol{e}_{i}^{*}\right\}, \frac{k}{\Gamma} \nu_{i} \nu_{j}\right):\left\|C^{*}-C\right\|_{\left\{\boldsymbol{e}_{i}\right\}}<\varepsilon\right\} .
$$

As a matter of notation, define $M\left[N_{\varepsilon}\right]$ for sufficiently small $\varepsilon$ by

$$
\begin{aligned}
M\left[N_{\varepsilon}\right]=\left\{\left(\left\{M_{i j} \boldsymbol{e}_{j}^{*}\right\},\right.\right. & \left.\frac{k}{\Gamma} \nu_{i} \nu_{j}\right):\left(M_{i j}\right) \in \mathcal{H}\left(k, \Gamma,\left\{\nu_{i}\right\}\right), \\
& \left.\left(\left\{\boldsymbol{e}_{i}^{*}\right\}, \frac{k}{\Gamma} \nu_{i} \nu_{j}\right) \in N_{\varepsilon}\left(\left\{\boldsymbol{e}_{i}\right\}, k, \Gamma,\left\{\nu_{i}\right\}\right)\right\}
\end{aligned}
$$

Now one can follow Ball and James [2], Cermelli and Mazzucco [4], Fosdick and Hertog [8] to prove (in particular) that if $\varepsilon$ is sufficiently small:

either $M\left[N_{\varepsilon}\right]=N_{\varepsilon}$, in which case $M_{i j} \boldsymbol{e}_{j}=Q \boldsymbol{e}_{i}$, with $Q$ orthogonal,

$$
\text { or } \quad M\left[N_{\varepsilon}\right] \cap N_{\varepsilon}=\emptyset .
$$


For the proof of (87), it suffices to remark that Ball and James' proof [2] of their Theorem 2.4 applies with the replacement of $\left(\mu_{i}^{j}\right) \in G L_{3}(\mathbb{Z})$ by $M=\left(M_{i j}\right)=\frac{1}{\Gamma}\left(m_{i j}\right) \in$ $\mathcal{H}\left(k, \Gamma,\left\{\nu_{i}\right\}\right)$ - this relies on the fact that if $\left\{M_{r}\right\}, r=1,2 \ldots$, is a convergent sequence of elements of $\mathcal{H}\left(k, \Gamma\left\{\nu_{i}\right\}\right)$, then $M_{r}=M_{r_{0}}$ if $r \geq r_{0}$, for some $r_{0} \in \mathbb{Z}$.

Definition Define $P\left(\left\{\boldsymbol{e}_{i}\right\}, k, \Gamma,\left\{\nu_{i}\right\}\right)$ by

$P\left(\left\{\boldsymbol{e}_{i}\right\}, k, \Gamma,\left\{\nu_{i}\right\}\right) \equiv\left\{Q \in O(3): Q \boldsymbol{e}_{i}=M_{i j} \boldsymbol{e}_{j}\right.$ for some $\left.\left(M_{i j}\right) \equiv M \in \mathcal{L}\left(k, \Gamma,\left\{\nu_{i}\right\}\right)\right\}$,

and call it the defective point group of the discrete structure defined by the parameters $\left(\left\{\boldsymbol{e}_{i}\right\}, k, \Gamma,\left\{\nu_{i}\right\}\right)$.

Proposition 10 If $\left(\left\{\boldsymbol{e}_{i}\right\}, k, \Gamma,\left\{\nu_{i}\right\}\right)$ and $\left(\left\{\boldsymbol{e}_{i}^{*}\right\}, k, \Gamma,\left\{\nu_{i}\right\}\right)$ define the same discrete structure, then the corresponding defective point groups are identical.

\section{Proof}

The hypotheses imply that, from (65)-(70),

$$
\boldsymbol{e}_{i}^{*}=M_{i j} \boldsymbol{e}_{j}, \quad \text { where } \quad\left(M_{i j}\right) \equiv M \in \mathcal{L}\left(k, \Gamma,\left\{\nu_{i}\right\}\right) .
$$

Let $Q \in P\left(\left\{\boldsymbol{e}_{i}\right\}, k, \Gamma,\left\{\nu_{i}\right\}\right)$, then $Q \boldsymbol{e}_{i}=\gamma_{i j} \boldsymbol{e}_{j}$ for some $\left(\gamma_{i j}\right)=\gamma \in \mathcal{L}\left(k, \Gamma,\left\{\nu_{i}\right\}\right)$. It follows that $Q \boldsymbol{e}_{i}^{*}=\left(M \gamma M^{-1}\right)_{i j} \boldsymbol{e}_{j}^{*}$. Since $\mathcal{L}\left(k, \Gamma,\left\{\nu_{i}\right\}\right)$ is a matrix group, one obtains that $Q \in P\left(\left\{\boldsymbol{e}_{i}^{*}\right\}, k, \Gamma,\left\{\nu_{i}\right\}\right)$ and this leads to the result.

$4.2 k \neq k^{*}, G_{\boldsymbol{c}} \neq G_{\boldsymbol{c}^{*}}$

Suppose now that $k \neq k^{*}$ so that the canonical bases $\boldsymbol{c}_{1}, \boldsymbol{c}_{2}, \boldsymbol{c}_{3}$ and $\boldsymbol{c}_{1}^{*}, \boldsymbol{c}_{2}^{*}, \boldsymbol{c}_{3}^{*}$ generate different groups $G_{\boldsymbol{c}}$ and $G_{\boldsymbol{c}^{*}}$ with $G_{\boldsymbol{c}} \neq G_{\boldsymbol{c}^{*}}$. Suppose however that $\left(\left\{\boldsymbol{c}_{i}\right\}, S_{p r}=k \delta_{p 3} \delta_{r 3}\right)$ and $\left(\left\{\boldsymbol{c}_{i}^{*}\right\}, S_{p r}^{*}=k^{*} \delta_{p 3} \delta_{r 3}\right)$ define the same set of points so that

$$
\begin{aligned}
w\left(\left\{\boldsymbol{e}_{i}\right\}, S_{p r}=\frac{\theta k}{\Gamma} \nu_{p} \nu_{r}\right) & =w\left(\left\{\boldsymbol{c}_{i}\right\}, S_{p r}=k \delta_{p 3} \delta_{r 3}\right) \\
& =w\left(\left\{\boldsymbol{c}_{i}^{*}\right\}, S_{p r}^{*}=k^{*} \delta_{p 3} \delta_{r 3}\right)=w\left(\left\{\boldsymbol{e}_{i}^{*}\right\}, S_{p r}^{*}=\frac{\theta^{*} k^{*}}{\Gamma^{*}} \nu_{p}^{*} \nu_{r}^{*}\right) .
\end{aligned}
$$

For this to occur it must be the case that $k$ and $k^{*}$ have the same parity ( $s o=\theta^{*}$ ) since the structure associated with $\left(\left\{\boldsymbol{c}_{i}\right\}, k \delta_{p 3} \delta_{r 3}\right)$ is a lattice for $k$ even or a multilattice for $k$ odd. In either case, $k$ and $k^{*}$ both even or both odd, the translation groups $T_{\boldsymbol{c}}$ and $T_{\boldsymbol{c}^{*}}$, given by (34), are lattices. If these lattices are to consist of the same points of $\mathbb{R}^{3}$, then if $k$ and $k^{*}$ are even,

$$
\left(\begin{array}{l}
c_{1}^{*} \\
c_{2}^{*} \\
c_{3}^{*}
\end{array}\right)=B\left(\begin{array}{l}
c_{1} \\
c_{2} \\
c_{3}
\end{array}\right)
$$

where $B \in G L_{3}(\mathbb{Z})$ and if $k$ and $k^{*}$ are odd then

$$
\left(\begin{array}{c}
\boldsymbol{c}_{1}^{*} \\
\boldsymbol{c}_{2}^{*} \\
\frac{1}{2} \boldsymbol{c}_{3}^{*}
\end{array}\right)=B\left(\begin{array}{c}
\boldsymbol{c}_{1} \\
\boldsymbol{c}_{2} \\
\frac{1}{2} \boldsymbol{c}_{3}
\end{array}\right)
$$


where $B \in G L_{3}(\mathbb{Z})$. Then the generators $\boldsymbol{e}_{1}, \boldsymbol{e}_{2}, \boldsymbol{e}_{3}$ of $G_{\boldsymbol{e}}$ and $\boldsymbol{e}_{1}^{*}, \boldsymbol{e}_{2}^{*}, \boldsymbol{e}_{3}^{*}$ of $G_{\boldsymbol{e}^{*}}$ are related in the following way. If $k$ is even then

$$
\left(\begin{array}{l}
\boldsymbol{e}_{1}^{*} \\
\boldsymbol{e}_{2}^{*} \\
\boldsymbol{e}_{3}^{*}
\end{array}\right)=\underbrace{\left(A^{*}\right)^{-1}\left(\begin{array}{ccc}
1 & 0 & -l_{0}^{*} \\
0 & 1 & -m_{0}^{*} \\
0 & 0 & \pm \Gamma^{*}
\end{array}\right) B\left(\begin{array}{ccc}
1 & 0 & -l_{0} \\
0 & 1 & -m_{0} \\
0 & 0 & \pm \Gamma
\end{array}\right)^{-1} A}_{\bar{M}}\left(\begin{array}{l}
e_{1} \\
\boldsymbol{e}_{2} \\
\boldsymbol{e}_{3}
\end{array}\right)
$$

or for $k$ odd,

$$
\left(\begin{array}{l}
\boldsymbol{e}_{1}^{*} \\
\boldsymbol{e}_{2}^{*} \\
\boldsymbol{e}_{3}^{*}
\end{array}\right)=\underbrace{\left(A^{*}\right)^{-1}\left(\begin{array}{ccc}
1 & 0 & -\left(2 l_{0}^{*}+\theta\left(\boldsymbol{l}^{*}\right)\right) \\
0 & 1 & -\left(2 m_{0}^{*}+\theta\left(\boldsymbol{m}^{*}\right)\right) \\
0 & 0 & \pm \Gamma^{*}
\end{array}\right) B\left(\begin{array}{ccc}
1 & 0 & -\left(2 l_{0}+\theta(\boldsymbol{l})\right) \\
0 & 1 & -\left(2 m_{0}+\theta(\boldsymbol{m})\right) \\
0 & 0 & \pm \Gamma
\end{array}\right)}_{\bar{M}} A\left(\begin{array}{l}
\boldsymbol{e}_{1} \\
\boldsymbol{e}_{2} \\
\boldsymbol{e}_{3}
\end{array}\right),
$$

where $A, A^{*}, B \in G L_{3}(\mathbb{Z})$.

Then (89) is to hold when $\left\{\boldsymbol{e}_{i}^{*}\right\}$ and $\left\{\boldsymbol{e}_{i}\right\}$ are related by (92), in the case $k$ and $k^{*}$ even, since (90) is sufficient that the points of $G_{\boldsymbol{e}}=T_{\boldsymbol{e}}=T_{\boldsymbol{c}}$ coincide with those of $G_{\boldsymbol{e}^{*}}=T_{\boldsymbol{e}^{*}}=T_{\boldsymbol{c}^{*}}$. However, (91) is not evidently sufficient to guarantee that the points of $G_{\boldsymbol{e}}$ coincide with those of $G_{\boldsymbol{e}^{*}}$ in the case where $\left\{\boldsymbol{e}_{i}^{*}\right\}$ and $\left\{\boldsymbol{e}_{i}\right\}$ are related by (93) and $k$ and $k^{*}$ odd, since one also requires that the cosets of $T_{\boldsymbol{e}}$ in $G_{\boldsymbol{c}}$ coincide with those of $T_{\boldsymbol{c}^{*}}$ in $G_{\boldsymbol{c}^{*}}$ (as sets of points in $\mathbb{R}^{3}$ ).

Proposition 11 In the case that $k$ and $k^{*}$ are both odd with $k \neq k^{*}$, the sets of points determined by the groups $G_{\boldsymbol{c}}, G_{\boldsymbol{c}^{*}}$ coincide provided that (91) holds and that certain conditions on the parity of the elements of $B$ are satisfied.

\section{Proof}

Relation (91) gives that the points of $T_{\boldsymbol{c}}$ and $T_{\boldsymbol{c}^{*}}$ coincide. Recall from (53) that the cosets of $G_{\boldsymbol{c}}$ are $T_{\boldsymbol{c}}, \boldsymbol{c}_{1} T_{\boldsymbol{c}}, \boldsymbol{c}_{2} T_{\boldsymbol{c}}, \boldsymbol{c}_{1} \boldsymbol{c}_{2} T_{\boldsymbol{c}}$ and similarly for $G_{\boldsymbol{c}^{*}}$. So we have to find the conditions that $\left\{\boldsymbol{c}_{1}, \boldsymbol{c}_{2}, \boldsymbol{c}_{1} \boldsymbol{c}_{2}\right\}$ are equivalent to $\left\{\boldsymbol{c}_{1}^{*}, \boldsymbol{c}_{2}^{*}, \boldsymbol{c}_{1}^{*} \boldsymbol{c}_{2}^{*}\right\} \bmod T_{\boldsymbol{c}}$ (recall Proposition 2(i)). So, as points of $\mathbb{R}^{3}, \boldsymbol{c}_{1}^{*}=\boldsymbol{c}_{1}^{\ell_{1}} \boldsymbol{c}_{2}^{\ell_{2}} \boldsymbol{c}_{3}^{\ell_{3}}=\ell_{1} \boldsymbol{c}_{1}+\ell_{2} \boldsymbol{c}_{2}+\left(2 \ell_{3}+k \ell_{1} \ell_{2}\right) \frac{1}{2} \boldsymbol{c}_{3}$, for some integers $\ell_{1}, \ell_{2}, \ell_{3}$. Note that $\boldsymbol{c}_{1}^{\ell_{1}} \boldsymbol{c}_{2}^{\ell_{2}} \boldsymbol{c}_{3}^{\ell_{3}}=\boldsymbol{c}_{1}^{L_{1}} \boldsymbol{c}_{2}^{L_{2}} \boldsymbol{c}_{3}^{L_{3}}, \bmod T_{\boldsymbol{c}}=\left\langle 2 \boldsymbol{c}_{1}, 2 \boldsymbol{c}_{2}, \boldsymbol{c}_{3}\right\rangle$ provided that $\bar{\ell}_{1}=\bar{L}_{1}, \bar{\ell}_{2}=\bar{L}_{2}$, where $\bar{\ell}=1$ if $\ell$ is odd, $\bar{\ell}=0$ if $\ell$ is even. Hence $\boldsymbol{c}_{1}^{*}=$ $\boldsymbol{c}_{1}^{\bar{\ell}_{1}} \boldsymbol{c}_{2}^{\bar{\ell}_{2}}, \boldsymbol{c}_{2}^{*}=\boldsymbol{c}_{1}^{\bar{m}_{1}} \boldsymbol{c}_{2}^{\bar{m}_{2}}, \bmod T_{\boldsymbol{c}}$, for some integers $m_{1}, m_{2}$. So $\left(\bar{\ell}_{1}, \bar{\ell}_{2}\right),\left(\bar{m}_{1}, \bar{m}_{2}\right)$ must be some two of $(1,0),(0,1),(1,1)$, and so $c_{1}^{*}=\bar{\ell}_{1} c_{1}+\bar{\ell}_{2} c_{2}+k \bar{\ell}_{1} \bar{\ell}_{2}\left(\frac{1}{2} c_{3}\right)=\bar{\ell}_{1} c_{1}+\bar{\ell}_{2} c_{2}+$ $\bar{\ell}_{1} \bar{\ell}_{2}\left(\frac{1}{2} c_{3}\right)$, mod $T_{\boldsymbol{c}}$, as $k$ is odd, and similarly for $\boldsymbol{c}_{2}^{*}$. Since $T_{\boldsymbol{c}}=\left\langle 2 \boldsymbol{c}_{1}, 2 \boldsymbol{c}_{2}, 2\left(\frac{1}{2} \boldsymbol{c}_{3}\right)\right\rangle$, these relations are constraints on the parity of elements in the first two rows of $B$ in (91). Next $\boldsymbol{c}_{1}^{*} \boldsymbol{c}_{2}^{*}=\boldsymbol{c}_{1}^{*}+\boldsymbol{c}_{2}^{*}+\frac{1}{2} k^{*} \boldsymbol{c}_{3}^{*}=\boldsymbol{c}_{1}^{*}+\boldsymbol{c}_{2}^{*}+\frac{1}{2} \boldsymbol{c}_{3}^{*}, \bmod T_{\boldsymbol{c}^{*}}$, as $k^{*}$ is odd, and there is a similar expression for $\boldsymbol{c}_{1} \boldsymbol{c}_{2}$. Since the elements of $\left\{\boldsymbol{c}_{1}^{*}, \boldsymbol{c}_{2}^{*}, \boldsymbol{c}_{1}^{*} \boldsymbol{c}_{2}^{*}\right\}$ are just $\left\{\boldsymbol{c}_{1}, \boldsymbol{c}_{2}, \boldsymbol{c}_{1} \boldsymbol{c}_{2}\right\}$, $\bmod T_{\boldsymbol{c}}$, it follows that

$$
\begin{aligned}
\frac{1}{2} \boldsymbol{c}_{3}^{*}=\boldsymbol{c}_{1}^{*} \boldsymbol{c}_{2}^{*}-\boldsymbol{c}_{1}^{*}-\boldsymbol{c}_{2}^{*}=\boldsymbol{c}_{1}^{*} \boldsymbol{c}_{2}^{*}+\boldsymbol{c}_{1}^{*}+\boldsymbol{c}_{2}^{*}= \\
\boldsymbol{c}_{1} \boldsymbol{c}_{2}+\boldsymbol{c}_{1}+\boldsymbol{c}_{2}=\boldsymbol{c}_{1} \boldsymbol{c}_{2}-\boldsymbol{c}_{1}-\boldsymbol{c}_{2}=\frac{1}{2} \boldsymbol{c}_{3}, \bmod T_{\boldsymbol{c}^{*}}=T_{\boldsymbol{c}} .
\end{aligned}
$$

Hence $\frac{1}{2} \boldsymbol{c}_{3}^{*}=\frac{1}{2} \boldsymbol{c}_{3}, \bmod T_{\boldsymbol{c}}$, and this is a constraint on the parity of elements in the third row of $B$ in (91). 
Finally, an argument which is in all essential details identical to that presented above in the case $k=k^{*}$ leads to an analysis of the Ericksen-Pitteri result (cf.(87)) in the case $k \neq k^{*}$. (For in the proof of the analogue of Proposition 8, one may note first of all that $\Gamma^{*}=\Gamma$ is obtained as before. The second paragraph of the proof may be replaced by the following:

'Next, since $\left\|\frac{k^{*}}{\Gamma} \nu_{i}^{*} \nu_{j}^{*}-\frac{k}{\Gamma} \nu_{i} \nu_{j}\right\| \geq \frac{1}{|\Gamma|}$ if $k^{*} \nu_{i}^{*} \nu_{j}^{*} \neq k \nu_{i} \nu_{j}$ for any choice of indices $i, j$, it follows that if $\left\|\frac{k^{*}}{T} \nu_{i}^{*} \nu_{j}^{*}-\frac{k}{T} \nu_{i} \nu_{j}\right\|$ is sufficiently small, then $k^{*}=k$ and $\nu_{i}^{*}= \pm \nu_{i}$, $i=1,2,3$, with the choice of sign possibly dependent on the index $i$.' The proof of Proposition 8 then proceeds as before, with the additional information that $k=k^{*}$. The calculations that follow Proposition 8 are taken across effectively unchanged (in this case $\left(\mu_{i}^{j}\right)$ is replaced by $\bar{M}$ from $\left.(92),(93)\right)$.

\section{Summary}

In the given class of defective crystals, when the dislocation density tensor $S$ has a given form, we have calculated the symmetries of energy density functions based on the assumptions that: (i) such functions depend only on the location of points in $\mathbb{R}^{3}$ which correspond to a certain discrete structure $G_{\boldsymbol{e}}$, and (ii) such functions are frame indifferent. The structures $G_{\boldsymbol{e}}$ are determined by $S$ and a choice of three vectors $\boldsymbol{e}_{1}, \boldsymbol{e}_{2}, \boldsymbol{e}_{3} \in \mathbb{R}^{3}$, and we have accounted for the fact that different choices of $\left(\left\{\boldsymbol{e}_{i}\right\}, S\right)$ may lead to the same set of points in $\mathbb{R}^{3}$.

The symmetries that are sufficiently small (in the sense of (74)) preserve the dislocation density tensor and extend uniquely to elastic deformations of a continuum in which the points of the crystal may be embedded. Indeed those 'elastic' (discrete) symmetries are conjugate to rotations which map the discrete structure to itself. The 'defective point group' which consists of those rotations that are conjugate to the elastic symmetries is an invariant of the discrete structures, once the dislocation density is chosen (cf. Proposition 10).

Acknowledgement

We thank the Engineering and Physical Sciences Research Council for support in the form of grant no EP/G047162/1.

\section{References}

1. L. Auslander, L. Green And F. Hahn, Flows on homogeneous spaces Annals of Mathematics Studies, Number 53, Princeton University Press, Princeton, New Jersey, 1963.

2. J. M. Ball And R. D. James, Proposed experimental tests of a theory of fine microstructure and the two-well problem, Phil. Trans. R. Soc. Lond. A 338, 1992, pp. 389-450.

3. N. Bourbaki, Elements of mathematics; General Topology, Hermann, Paris, 1966.

4. P. Cermelli and E. Mazzucco, A note on the model of crystaline defects in EricksenPitteri neighbourhoods, Physica D: Nonlinear Phenomena, 99, 1996, 350-358.

5. P. Cermelli AND G. P. PARRY, The structure of uniform discrete defective crystals, Continuum Mech. Thermodyn., 18, 2006, 47-61.

6. C. Davini, A proposal for a continuum theory of defective crystals. Arch. Rational Mech. Anal., 96, 1986, 295-317. 
7. J. L. ERICKSEN, On groups occurring in the theory of crystal multi-lattices, Arch. Rat. Mech. Anal., 148, 1999, pp. 145-178.

8. R. L. Fosdick And B. Hertog, Material symmetry and crystals, Arch. Rational Mech. Anal., 117, 1990, pp 43-72.

9. P. Hall, A contribution to the theory of groups of prime power order, Proc. London. Math. Soc., Ser 2, 36, 1933, pp. 29-95.

10. A. MAL'CEV, On a class of homogeneous spaces, Isvestiya Akad. Nauk SSSR Ser., Mat 13, pp 9-32. Am. Math. Soc. Translation 39, 1949.

11. G. P. PARry AND M. S̆IlhavÝ, Elastic scalar invariants in the theory of defectivve crystals, Proc. R. Soc. Lond. A, 455, 1999, pp. 4333-4346.

12. G. P. PARRY, Group properties of defective crystal structures, Mathematics and Mechanics of Solids, 8, 2003, 515-537.

13. G. P. PARRY, Symmetries of continuously defective crystals, in Mathematical modelling of bodies with complicated bulk and boundary behaviour, ed. M. Šilhavý, Quaderni di Mathematica 20, Dipartimento di Matematica della Seconda Università di Napoli, 2007, $135-158$.

14. G. P. PARRY, Rotational symmetries of crystals with defects, J. Elast. 94, 2009, 147-166.

15. G. P. PARrY, Elastic symmetries of deffective crystals, J. Elast. 101, 2010, 101-120.

16. M. PitTERI, Reconciliation of local and global symmetries of crystals, Journal of Elasticity 14, 1984, 175-190

17. M. Pitteri And G. Zanzotto, Continuum models for phase transitions and twinning in crystals, Chapman and Hall/CRC, Boca Raton, London, New York, Washington D.C., 2003.

18. W. Thurston, Three dimensional geometry and topology, vol. 1, Princeton University Press, Princeton, New Jersey, 1997. 